\title{
Comparing albino and hooded Wistar rats of both sexes on a range of behavioral and learning tasks
}

\author{
R. A. BOAKES, B. BOOT, J. V. CLARKE, and A. CARVER \\ University of Sydney, Sydney, Australia
}

\begin{abstract}
Albino and hooded Wistar rats of both sexes were compared on several behavioral measures and learning tasks. Overall the most marked behavioral differences produced by albinism were reduced metabolism - as indicated by food and water consumption, defecation, and direct calorimetric measures of respiratory quotient and energy expenditure - and emotionality_as displayed in an open field, plus maze, and radial maze. No sex or strain differences were found for neophobia or preference for saccharin, but less tolerance of bitter (quinine) was shown by hooded rats and less tolerance of sour $(\mathrm{HCl})$ by males. In learning tasks in restricted environments, comparable acquisition across groups was found for instrumental conditioning, appetitive classical conditioning, and taste aversion learning. However, hooded rats showed greater latent inhibition with auditory stimuli and more sensitivity to contextual cues, both in nausea-based conditioning and in the radial maze. Overall the results suggest that careful choice of rat strain is required in behavioral experiments where environmental factors are critical.
\end{abstract}

For reasons that appear to be mainly historical or practical, behavioral laboratories across the world differ in the strains of rats they use. There are few data allowing a researcher an informed choice of strain to employ in a particular kind of study. An exception is the case of experiments involving visual discriminations where the visual defects of albino rats make them unsuitable (e.g., Birch \& Jacobs, 1979; Lund, Lund, \& Wise, 1974). The visual superiority of pigmented rats appears to have encouraged the first use of hooded strains in behavioral research (Lashley, 1930) when previously only albino rats had been used. Recent studies where the performance of albinos has been found to be worse than that of pigmented strains have included delayed-nonmatching-to-sample (Aggleton, 1996) and Morris water maze (Tonkiss, Shultz, \& Galler, 1992).

Albino rats may be anomalous in ways other than just impaired visual ability. Comparisons between albino and pigmented rats have documented various differences in sensory-neural systems, endocrine function, and biochemistry, which are consistent with the possibility that failure to synthesize melanin has widespread consequences for the physical development and present functioning of an individual organism (Creel, 1980). Possible behavioral consequences of such physical differences do not, however, appear to have been tested by tasks that are unaffected by visual stimuli (see Andrews, Jansen, Linders, Princen, \& Broekkamp, 1995). Thus, the main aim of the present study

This research was partly supported by the Australian Research Council. We are grateful to Iain McGregor for the loan of the plus maze used in Experiment 2 and for technical advice, to Dale Atrens for use of the metabolic chamber in Experiment 3, and to John Holden for assistance with this equipment. Correspondence should be addressed to $\mathrm{R}$. A. Boakes, Department of Psychology, University of Sydney, NSW 2006, Sydney, Australia (e-mail: bobb@psych.usyd.edu.au). was to investigate whether albino and hooded rats might differ in ways that could affect the outcomes of behavioral experiments, other than those involving visual discriminations. We used Wistar rats, since albinos of this strain are used on a large scale worldwide for behavioral research and a hooded variety was available. Use of a single strain meant that any obtained behavioral differences were more likely to reflect the effect of albinism per se than when, as has been common in other studies (e.g., Aggleton, 1996; Andrews et al.,1995), pigmented and albino rats from different strains are compared.

The common strategy in between-strain research has been to compare several groups of naive animals on a restricted set of measures. As a result, meaningful comparisons across tests, usually carried out in different laboratories and with animals raised under varying conditions, are difficult to make. The different experimental strategy adopted here was to compare the same groups of rats on a wide range of tests. The obvious drawback to this approach is that comparisons across different tests are confounded with age and weight changes, as well as with changes in subjects' previous experience. To meet this problem, the present study included sex differences as a factor. These are far more extensively documented than strain differences (see, e.g., Gray, 1987; Johnston \& File, 1991). We reasoned that, if the sex differences detected in our study corresponded to those found with naive animals of varying ages, it was less likely that any strain differences we detected could have been greatly affected by the age and past history of our subjects.

A major concern of this study was the extent to which possible differences on behavioral measures are related to performance on a variety of learning tasks. Consequently, as shown in Table 1, subjects were tested on a number of behavioral measures before being given a series of learning tasks that were chosen to provide as wide a range as 
Table 1

Summary of Procedures and Main Findings

With Respect to Strain and Sex Differences

\begin{tabular}{|c|c|c|c|c|c|}
\hline & Experiment & Week & Behavior & $\begin{array}{c}\text { Albino } \\
\text { Versus Hooded }\end{array}$ & $\begin{array}{c}\text { Female } \\
\text { Versus Male }\end{array}$ \\
\hline & $\begin{array}{l}\text { Running wheel } \\
\text { Open field }\end{array}$ & 1 & $\begin{array}{l}\text { Running, } \\
\text { line crossing, } \\
\text { defecation }\end{array}$ & $\begin{array}{l}\text { n.s. } \\
a>h \\
a \ll h\end{array}$ & $\begin{array}{l}\mathrm{f}>\mathrm{m} \\
\mathrm{f}>\mathrm{m} \\
\mathrm{f}<\mathrm{m}\end{array}$ \\
\hline 2 & Plus maze & 3 & Time in open & $a>h$ & $\mathrm{f}>\mathrm{m}$ \\
\hline 3 & Metabolic rate & 33 & $\begin{array}{l}\text { Activity, } \\
\text { energy expenditure, } \\
\text { respiratory quotient }\end{array}$ & $\begin{array}{l}a>h \\
a<h \\
a>h\end{array}$ & $\begin{array}{l}\text { n.s. } \\
f>m \\
f>m\end{array}$ \\
\hline 4 & $\begin{array}{l}\text { Taste neophobia } \\
\text { and preferences }\end{array}$ & 6 & $\begin{array}{l}\text { Saccharin neophobia, } \\
\text { saccharin preference, } \\
\text { sour preference, } \\
\text { bitter preference }\end{array}$ & $\begin{array}{l}\text { n.s. } \\
\text { n.s. } \\
\text { n.s. } \\
\mathrm{a}>\mathrm{h}\end{array}$ & $\begin{array}{l}\text { n.s. } \\
\text { n.s. } \\
\text { f }>\mathrm{m} \\
\text { n.s. }\end{array}$ \\
\hline 5 & $\begin{array}{l}\text { Instrumental } \\
\text { conditioning and } \\
\text { incentive learning }\end{array}$ & 7 & $\begin{array}{l}\text { Neophobia, } \\
\text { acquisition of leverpress, } \\
\text { response rate }\end{array}$ & $\begin{array}{l}\mathrm{a} \ll \mathrm{h} \\
\text { n.s. } \\
\mathrm{a}<\mathrm{h}\end{array}$ & $\begin{array}{l}\text { n.s. } \\
\text { n.s. } \\
\text { f }<\mathrm{m}\end{array}$ \\
\hline 6 & $\begin{array}{l}\text { Classical } \\
\text { conditioning and } \\
\text { latent inhibition }\end{array}$ & 15 & $\begin{array}{l}\text { Acquisition, } \\
\text { response rate, } \\
\text { latent inhibition }\end{array}$ & $\begin{array}{l}\text { n.s. } \\
\text { n.s. } \\
\mathrm{a}<\mathrm{h}\end{array}$ & $\begin{array}{l}\text { n.s. } \\
\text { n.s. } \\
\text { n.s. }\end{array}$ \\
\hline 7 & $\begin{array}{l}\text { Nausea-based } \\
\text { conditioning }\end{array}$ & $\begin{array}{l}19, \\
25\end{array}$ & $\begin{array}{l}\text { Taste aversion, } \\
\text { context aversion }\end{array}$ & $\begin{array}{l}\text { n.s. } \\
\mathrm{a}<\mathrm{h}\end{array}$ & $\begin{array}{l}\mathrm{f}<\mathrm{m} \\
\mathrm{f}<\mathrm{m}\end{array}$ \\
\hline 8 & $\begin{array}{l}\text { Spatial learning } \\
\text { (radial maze) }\end{array}$ & 31 & $\begin{array}{l}\text { Defecation, } \\
\text { neophobia, } \\
\text { working memory, } \\
\text { reference memory }\end{array}$ & $\begin{array}{l}a \ll h \\
a \ll h \\
\text { n.s. } \\
\text { n.s. }\end{array}$ & $\begin{array}{l}\mathrm{f}<\mathrm{m} \\
\mathrm{f}<\mathrm{m} \\
\text { n.s. } \\
\text { n.s. }\end{array}$ \\
\hline
\end{tabular}

Note - "Week" refers to when an experiment started. At the start of Week I, all hooded $(h)$ rats were 55 days old, the male $(\mathrm{m})$ albinos (a) were 67 days old, and the female (f) albinos were 74 days old. See text for details of measures and explanation of abbreviations.

possible given the availability of equipment. The experiments were designed to allow strain and sex comparisons both on simple learning tasks, as listed in Table 1, and in more subtle phenomena. Given the absence of previous comparisons of this kind, the strategy adopted was to aim for breadth, rather than concentrate on a particular aspect of learned behavior.

\section{GENERAL METHOD}

\section{Subjects}

The subjects used throughout included 32 hooded Wistar rats, bred at the University of Sydney from stock purchased from the University of Adelaide, and born on March 8 . The remaining 32 were naive albino Wistars, bred at CULAS, N.S.W., from an outbred strain derived from stock at ANSTO (Lucas Heights, N.S.W.) in 1964, and born on February 17 (females) and February 24 (males). There were 16 males and 16 females from each strain. Throughout the study subjects were housed communally as unchanged singlesex groups of 8 in large white translucent polyethylene cages, $40 \times$ $63 \times 20 \mathrm{~cm}$, in the colony room. Lighting in this room was on a $12-$ $\mathrm{h}$ light:dark cycle with onset at $0700 \mathrm{~h}$ and offset at $1900 \mathrm{~h}$. Except where specified below, unrestricted access to laboratory chow and water was allowed in the home cages throughout the study.

Rats were handled and weighed throughout the 8 months of the study, commencing April 30 (Week 0), when all rats were given 2-3 min individual handling for 3 successive days. Care was taken to give equivalent treatment to subjects in this and all other respects. Body weights are shown in Figure 1, which indicates the normal heavier weight and more rapid growth of males. The albinos weighed more than the hooded rats. For the first few weeks this presumably reflected mainly the fact that the albinos were $2-3$ weeks older, but this cannot account for the larger differences that developed as the study progressed. Table 1 gives the week each experiment was begun so that the weights of subjects at that time can be derived from Figure 1.

All experiments incorporated a two-factor between-subjects design with strain and sex as factors, and included complete counterbalancing.

\section{Experiment 1 \\ Running and Open Field Measures}

Although a few studies have looked for effects of albinism on activity in mice (DeFries, Hegmann, \& Weir, 1966; Katz \& Doyle, 1981; Ward \& Lariviere, 1980), a possible connection between albinism and activity in rats has hardly been studied. Some indication that hoodeds are more active than albinos comes from a breeding study selecting for high levels of rearing (Saunders, 1981). More standard measures were used here: running wheels to measure general activity and the open field test (Gray, 1987; C. S. Hall, 1936; Van der Staay, Kerbusch, \& Raaijmakers, 1990) to measure exploratory behavior and emotionality. Because time of day affects activity level (see, e.g., Mistlberger, 1993) and could interact with strain or sex differences, the comparisons were carried out at two different times of day.

\section{Method}

Apparatus. Four running wheels with sheet metal sides and steel rod floors were used, $11-\mathrm{cm}$ wide $\times 126-\mathrm{cm}$ circumference. The open field apparatus (Broadhurst, 1960, p. 32) was $86.5 \mathrm{~cm}$ in di- 


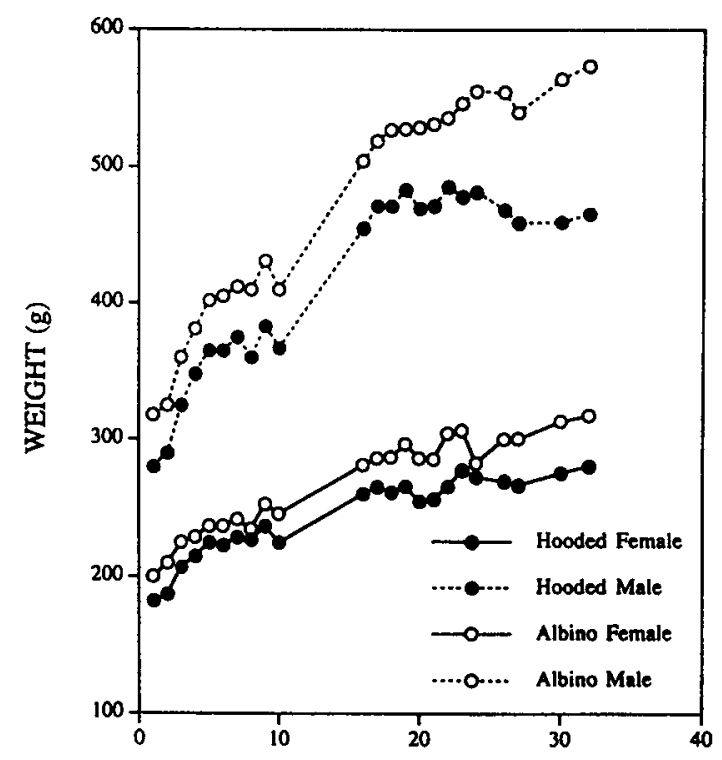

WEEKS FROM START OF EXPERIMENTS

Figure 1. Mean body weights of the four groups of rats $(n=16)$ over the series of experiments. See Table 1 for when each experiment started.

ameter with 20-cm-high metal boundary walls. The floor area was marked out into 19 sections radiating from the center. Illumination was provided by ceiling-mounted fluorescent lights on the far side of the laboratory so that the open field was only dimly lit.

Procedure. In each of five daily sessions, 4 rats from each homecage squad of 8 were first placed in the running wheels for $15 \mathrm{~min}$, while the other 4 were tested individually for 2 min each in the open field. These rats were then placed in the wheels for a $15-\mathrm{min}$ period, while the first 4 were given their 2 -min open field tests. Half the subjects in each group were tested in the morning, 0700-1000 h, and half were tested in the evening, $1900-2200 \mathrm{~h}$. The number of boluses produced by each rat was recorded for each session in the open field.

\section{Results}

The main findings from this experiment were that in the wheels, albino males ran less than hooded males, whereas albino females ran more than hooded females, and in the open field, albinos made more line crossings than hooded rats, independently of sex. As for sex differences, activity scores for females were higher than for males on both measures.

Running wheels. Daily wheel turns, shown in Figure 2, were submitted to a mixed analysis of variance (ANOVA) with sex, strain, and time of day as between-subjects factors and session as the within-subjects factor. This indicated that (1) female rats ran more than males $[F(1,56)=$ $52.86, p<.0001] ;(2)$ there was no detectable main effect of strain $(F<1.2) ;(3)$ there was an interaction between strain and $\operatorname{sex}[F(1,56)=7.35, p<.05]$, which, from an analysis of simple effects, indicated that among males, hoodeds ran more than albinos $(p<.005)$, but, among females, hoodeds ran less than albinos $(p<.001)$; (4) there was an interaction between sex and time of day $[F(1,56)=$ $7.62, p<.01]$, which inspection of the figure indicated that female, but not male, rats ran more in the evening than in the morning; (5) running increased over sessions $[F(4,224)=44.99, p<.0001]$; and $(6)$ this increase was greater for females $[F(4,224)=6.83, p<.001]$. No other interactions were significant.

Open field. Line crossings, shown in Figure 3, were submitted to a mixed ANOVA using the same factors for running. This indicated that (1) the females made more
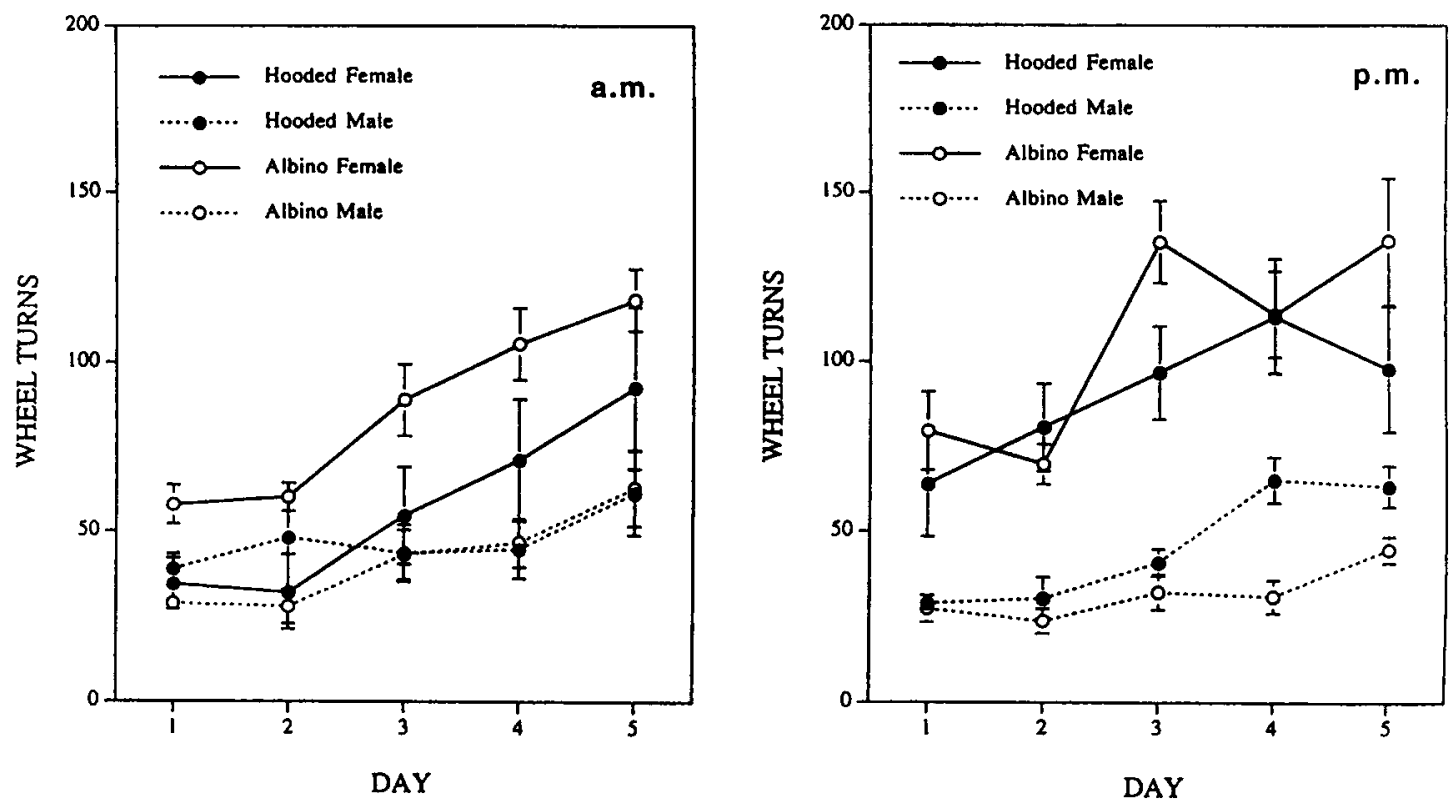

Figure 2. Experiment 1: Running wheel. Mean number, plus standard errors of the mean, of wheel turns during a 15-min session. Data from groups tested in the morning $(n=8)$ are shown in the left panel and data from groups tested in the evening $(n=8)$ in the right panel. 

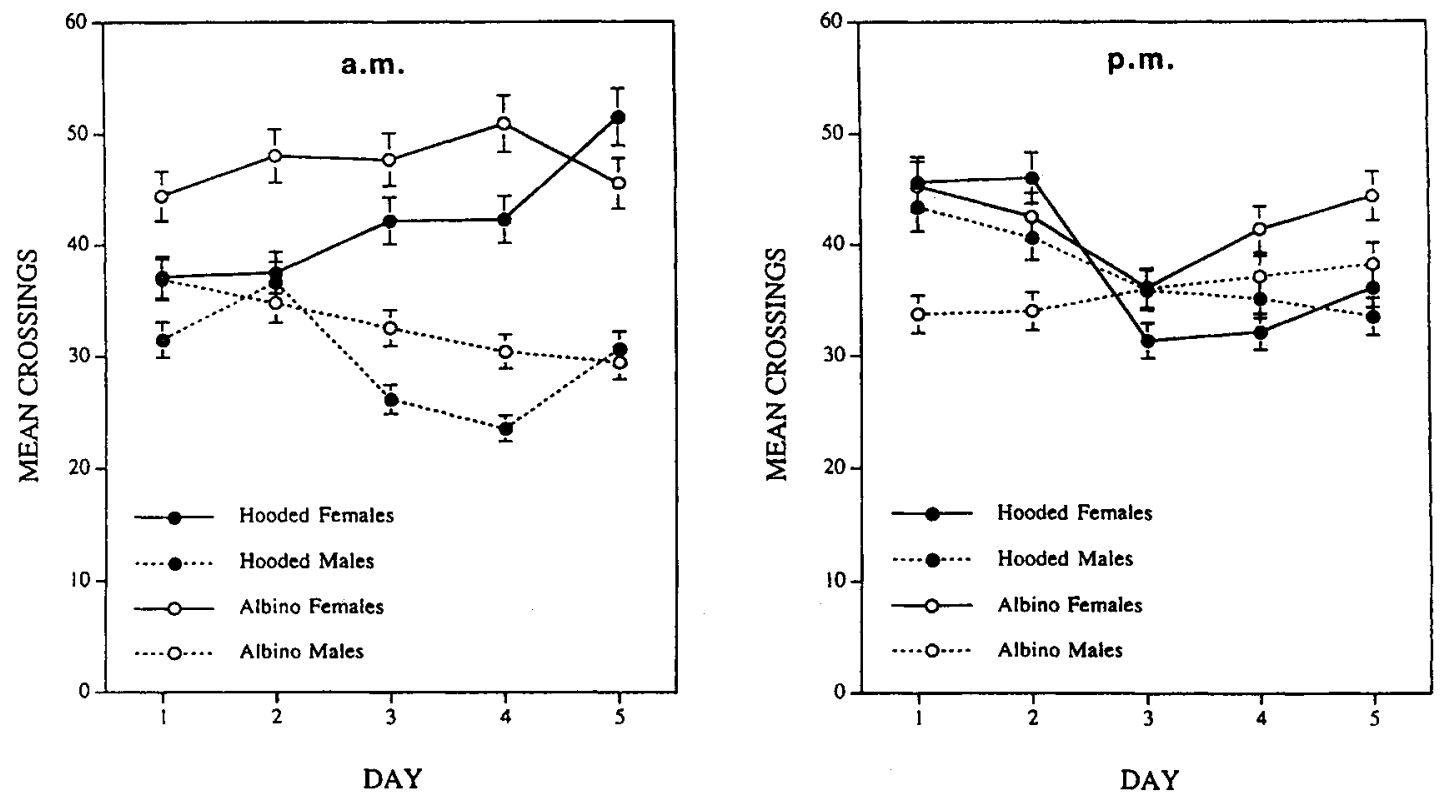

Figure 3. Experiment 1 : Open field. Mean number, plus standard errors of the mean, of line crossings during a 2-min session in the open field maze. Data from groups run in the morning $(n=8)$ are shown in the left panel and data from evening groups $(n=8)$ in the right panel.

crossings than males $[F(1,56)=31.29, p<.0001] ;(2)$ albinos made more crossings than hooded rats $[F(1,56)=$ $2.82, p<.01] ;(3)$ there was no significant main effect of time of day $(F<1)$; (4) males' scores were higher in the evening than in the morning, whereas females' scores were higher in the morning $[F(1,56)=11.41, p<.005]$; and (5) line crossing decreased over the five sessions $[F(4,224)=2.62, p<.05]$. No other significant interactions were detected.

The most striking finding from the bolus counts was that no albino defecated in any of the five sessions, whereas all the hoodeds did. For each of the latter animals, the mean number of boluses per session was calculated, and these data were submitted to a $2 \times 2$ ANOVA with sex and time of day as factors. This revealed that the males, with a mean of 2.1 boluses per 2-min session, defecated more than the females, whose mean was 1.6 per session $[F(1,28)=5.23, p<.05]$. No effect or interaction involving time of day was detected $(p s>.10)$.

\section{Discussion}

The procedures used in this experiment proved sufficiently sensitive to detect the sex differences in activity level that have been reported previously (e.g., Gray, 1987), in that females of both strains ran more in the wheels and made more crossings in the open field than their male counterparts. In contrast, a main effect of strain was found only in the open field. This measure is more likely to confound activity levels with anxiety. The high bolus count for the hoodeds, in the absence of any defecation by the albinos, suggests that the anxiety level for hoodeds was indeed much higher. (See below for defecation in the home cage.) This would appear to account for their lower rate of crossing and the apparent discrepancy with the running wheel measure, where no strain difference was found.

\section{Experiment 2 Elevated Plus Maze}

Reluctance to enter the open arms of a plus maze is a widely used measure of anxiety (e.g., Pellow, Chopin, File, \& Briley, 1985). Maudsley Reactive rats, the strain selectively bred for low ambulation and high defecation in the open field, show greater aversion for open arms than Maudsley Nonreactive and Wistar albino controls (Overstreet, Rezvani, \& Janowsky, 1992). Following such reports and the outcome of Experiment 1, it was predicted that open-arm preference would be (1) greater in females than in males, thus confirming the welldocumented greater anxiety in male rats (see, e.g., Gray, 1987), and (2) following Experiment 1, greater anxiety in albinos than in hooded animals.

\section{Method}

Apparatus. The plus maze consisted of two open arms and two enclosed arms, each arm $50 \mathrm{~cm}$ in length, at a height of $53 \mathrm{~cm}$ above the floor of a large and brightly illuminated laboratory. The two open arms were opposite each other. In the center was an area of $10 \mathrm{~cm}^{2}$

Procedure. Subjects were given a single 5-min session, which began with placement in the center of the maze facing an open arm. Time on either of the open arms was recorded; this was defined as 


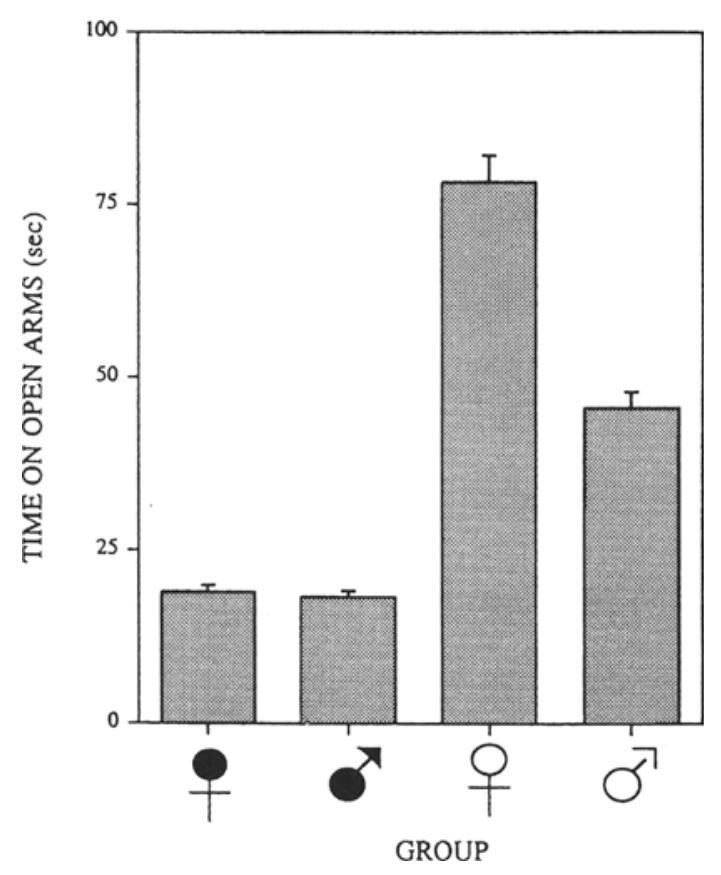

Figure 4. Experiment 2: Plus maze. Mean time, plus standard errors of the mean, spent on the open arms of the elevated plus maze during a single 15-min session. Filled symbols indicate data from the hooded groups and open symbols those from the albino groups.

all four feet over the line marking off the arm from the center area. The maze was wiped with a solution of $1 \%$ vinegar in water before and after each session to remove odors.

\section{Results}

An ANOVA with sex and strain as factors, as well as inspection of the data shown in Figure 4, indicated that (1) albinos spent more time on the open arms than hoodeds $[F(1,60)=59.11, p<.0001],(2)$ females spent more time on the open arm than males $[F(1,60)=8.75, p<$ $.005]$, but (3) a significant interaction between strain and sex $[F(1,60)=8.05, p<.01]$ indicated that this sex difference was larger in albino rats.

\section{Discussion}

These results complement the defecation data from Experiment 1 . Both measures indicated a higher level of emotionality in hooded animals. In Experiment 1, hooded males defecated more than hooded females, but no comparable sex difference in albinos could be detected because neither produced any boluses. In the second experiment, albino males spent less time on the open arms than albino females, but no such sex difference was detectable in hooded animals, arguably because both sexes displayed a high level of fear. Thus, the data from the two experiments are consistent with the general claims that hooded rats are more emotional than albinos and that males are more emotional than females.
Nonetheless, this conclusion needs to be treated with some caution. In the case of the plus maze, anxiety is presumably generated by perceiving the height of the open arm above the floor. Albinos' impaired vision may mean that they are less anxious because they are less able to perceive the danger. In the case of bolus counts, this measure must also be affected by body size and food intake.

Given the lack of defecation during experimental sessions by the albinos, we decided to monitor food intake and waste production of the animals in their home cages immediately following the above experiment. Over a 3day period of unrestricted food and water, when the animals were otherwise undisturbed, the number of boluses in each cage was counted daily and the amount of food consumed on the 1 st and 3 rd days was monitored. The females ate less and produced fewer boluses, as expected from their smaller body size. Among the hooded rats, the daily food intake of an individual male averaged $28.9 \mathrm{~g}$, with a mean of 6.9 boluses, while the food intake of females averaged $19.8 \mathrm{~g}$, with a mean of 5.6 boluses. Among the albino rats, the daily food intake of males averaged $25.2 \mathrm{~g}$, with a bolus count of 6.2 , while the food intake of females averaged $16.4 \mathrm{~g}$, with a bolus count of 4.9 . These measures were from home-cage groups of 8 animals. Thus, no individual data could be obtained for statistical analysis of the above differences. Nonetheless these differences were consistent over the small number of days studied and suggested an interesting possibility with respect to strain differences. For each of the measurements, food intake was higher for hoodeds than for albinos of the same sex, as was defecation. At this time body weight differences between the two strains were small, particularly for females, but the tendency was for albinos to weigh more than hoodeds (Figure 1). This suggested that metabolic rate was higher for the hoodeds than for the albinos. This was tested in Experiment 3.

\section{Experiment 3 Metabolic Rate}

Lack of access to functioning equipment meant delaying this experiment until the others had been completed. The method used to examine possible metabolic differences employed an open-circuit calorimeter, which monitors the rate of carbon dioxide production relative to oxygen consumption. The equipment also measured activity in terms of fine movement. This contrasted with the gross measures of activity, running, and line crossing used in Experiment 1. The custom-built calorimeter has been used extensively at the University of Sydney to investigate the effect of pharmacological substances on metabolic rate (e.g., Atrens \& Menendez, 1993).

\section{Method}

Subjects. Only 1 squad from each of the two sex-strain homecage squads was used. Thus, there were 8 albino female cage-mates, 8 albino males, 8 hooded females, and 8 hooded males. They were 
9 months old at this time, when the weights were as shown in Figure 1. They had unrestricted access to food and water in their home cages, except for a period of $2 \mathrm{~h}$ prior to the daily experimental sessions when food and water were removed from these cages.

Apparatus. Respiratory quotient (RQ) and energy expenditure (EE) were calculated after oxygen $\left(\mathrm{O}_{2}\right)$ consumption and carbon dioxide $\left(\mathrm{CO}_{2}\right)$ production were recorded in an open-circuit calorimeter. Two clear acrylic cylindrical chambers with stainless steel grid floors and a volume of 6.281 were used, one for testing the rats and the other as a reference standard for calibration. Compressed atmospheric air at a flow rate of $1,600 \mathrm{ml} / \mathrm{min}$ and a pressure of $8 \mathrm{kPa}$ above atmospheric was continuously drawn through both chambers. Air leaving one of the chambers was split and directed for analysis, while air from the other chamber was exhausted to the atmosphere. A sample of $110 \mathrm{ml} / \mathrm{min}$ was directed through a Perma Pure (Toms River, NJ) permeation drier (Model PD750-12PP) and a CD-3a $\mathrm{CO}_{2}$ analyzer (Applied Electrochemistry, Sydney). The analyzers were calibrated daily with primary gravimetric standards (Commonwealth Industrial Gases, Sydney).

Locomotor activity was recorded by placing the test chamber on an electronic balance (Mettler PE-2000) and using the unintegrated signal from the strain gauge. The reliability and validity of this method have been demonstrated in other studies (Atrens \& Menendez, 1993). A microcomputer system controlled and monitored the calorimeter. The computer provided minute-by-minute records of air flow, $\mathrm{CO}_{2}$ production, $\mathrm{O}_{2}$ consumption, and activity counts. The following calculations were made: $\mathrm{EE}(\mathrm{kJ})=\mathrm{mol} \mathrm{O}_{2}$ $(364+113 R Q) ; \mathrm{RQ}=$ vol. $\mathrm{CO}_{2}$ produced/vol. $\mathrm{O}_{2}$ consumed. Energy expenditure was expressed in joules/gram to allow for different body weights.

Procedure. On Days 1 and 2, each rat was habituated to the metabolic apparatus for a $15-\mathrm{min}$ period. On Day 3, each rat was placed in the calorimeter for $18 \mathrm{~min}$ and recordings were made each minute. The first $3 \mathrm{~min}$ were used for calibration and excluded from analysis. Owing to equipment failure activity, data from 2 subjects were lost.

\section{Results}

Activity steadily declined during the session, as normally found with this equipment (e.g., Atrens \& Menen- dez, 1993). As seen in the left-hand panel of Figure 5, albinos were more active than hoodeds and, although there was no overall sex difference, among the albinos, males were more active than females. A mixed ANOVA with sex and strain as between-subjects factors and time as a repeated measure revealed a main effect of time during session $[F(14,390)=2.15, p<.001]$, a main effect of strain $[F(1,390)=111.96, p<.0001]$, no main effect of sex $[F(1,390)=1.66]$, and an interaction between strain and $\operatorname{sex}[F(1,390)=4.36, p<.05]$. No other interactions were significant.

The EE measure provides an index of metabolic rate. This decreased steadily over the session, as is normal for such tests. As indicated in the middle panel of Figure 5, EE was higher in hoodeds than in albinos, and higher in females than in males. The difference between hoodeds and albinos was greater in females. A mixed ANOVA applied to the EE data revealed a main effect of time $[F(1,420)=3.89, p<.01]$, a main effect of strain $[F(1,420)$ $=432.31, p<.0001]$, a main effect of $\operatorname{sex}[F(1,420)=$ $739.47, p<.0001]$, and an interaction of strain and sex $[F(1,420)=339.87, p<.0001]$. No other interactions were significant.

Relative utilization of carbohydrate and fatty tissue is indicated by the $R Q$, with high values indicating high use of carbohydrate and consequent sparing of fat. No change was detected across the session, as is normal (e.g., Atrens \& Menendez, 1993). As shown in the righthand panel of Figure 5, RQ was higher in albinos than in hooded rats, and higher in females than in males. Furthermore, there was an interaction whereby the difference between females and males was greater for albinos than for hooded rats. A mixed ANOVA applied to these RQ scores revealed a main effect of strain $[F(1,420)=$ $86.90, p<.001]$, a main effect of $\operatorname{sex}[F(1,420)=9.72$,
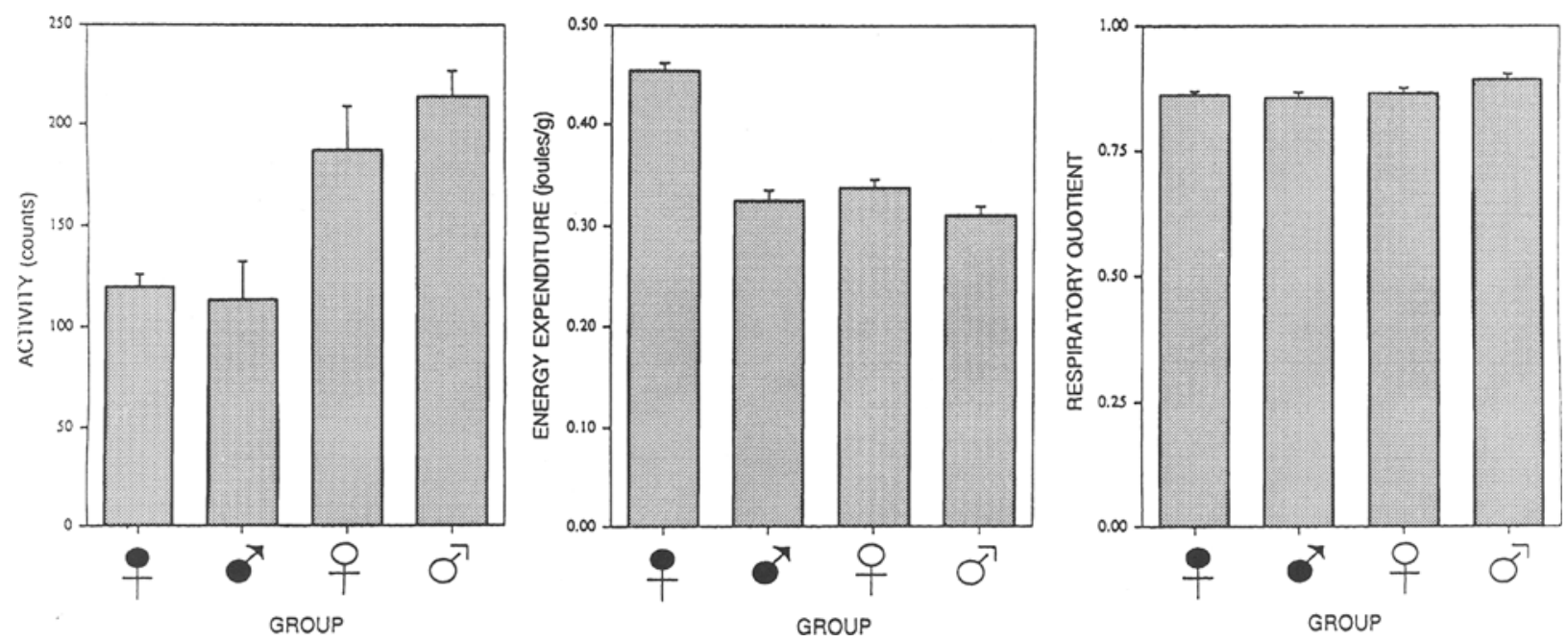

Figure 5. Experiment 3: Metabolic measures. Data from the 15-min period in the metabolic chamber showing mean, plus standard errors of the mean, of activity count (left panel), energy expenditure (middle panel), and respiratory quotient (right panel) for the four groups. Filled symbols indicate data from the hooded groups and open symbols those from the albino groups. 
$p<.005]$, and a strain $\times$ sex interaction $[F(1,420)=$ $57.638, p<.0001]$. There was no detectable change in scores over time $(F<1)$.

\section{Discussion}

The major result was the finding that EE in the hooded rats was higher than in albinos, thus confirming the suggestion from the previous data on food intake and bolus counts that the resting metabolic rate of hoodeds is greater. The finding that RQ was higher in albinos than in hooded rats is consistent with the observation that, over the course of the study, body weight increase (Figure 1), to which accumulation of fatty tissue makes a major contribution, was slower in hooded than in albino rats.

The major implication of the activity data with respect to metabolism is that the lower activity counts given by the hooded animals rule out the possibility that their EE levels were higher because they were more active.

\section{Experiment 4}

\section{Taste Preferences and Taste Neophobia}

Nachman (1959) found a tendency for hooded rats to show a greater preference for saccharin than albinos. This was later related by Bartoshuk (1979) to individual differences in ability to taste the bitter component of saccharin, $6-n$-propylthiouracil. There appear to have been no other attempts to find strain differences of this kind. As for sex differences, Hamilton and Timmons (1976) reported greater sensitivity to taste properties in females.

The present experiment examined possible strain and sex differences in taste neophobia and taste preferences. Rats given a novel taste such as quinine-adulterated water (Dess \& Chapman, 1990) or saccharin (Dess, 1992) show considerable individual variation in degree of suppression of drinking. Furthermore, "finickiness" toward quinineadulterated water can be sensitive to prior stress (see, e.g., Dess \& Chapman, 1990). If the differences in anxiety found between the groups in Experiment 1 partly reflect differences in fearfulness in a new environment, the question arises as to whether the same pattern would be found for taste neophobia: Do males show greater neophobia than females, and hoodeds greater neophobia than albinos?

Preference for sour was tested in a pilot experiment carried out in the home cages. After all subjects were placed on a 23-h water deprivation schedule, four 15 -min two-bottle tests within a 70 -min session were given on 2 successive days. On the 1st day, the choice was between water and $0.005 \mathrm{M} \mathrm{HCl}$, and on the 2nd day, it was between water and $0.01 \mathrm{M} \mathrm{HCl}$. The results suggested that males show greater avoidance of sour tastes than females. Group testing meant that the reliability of this result could not be evaluated, but it did suggest that the present experiment, employing individual testing, should examine a wider range of substances than just quinine (bitter) or saccharin.

\section{Method}

Apparatus. A laboratory separate from the colony room contained a set of eight individual drinking chambers, $20 \times 30 \times$
$15 \mathrm{~cm}$. They were of transparent acrylic but were visually isolated from each other. Glass drinking bottles $(200 \mathrm{ml})$, with stainless steel spouts containing ball bearings, could be inserted at an angle of approximately $45^{\circ}$ through the wire mesh top to each cage. When two bottles were inserted for choice tests, their spouts were $10 \mathrm{~cm}$ apart.

Procedure. The water deprivation schedule established for the pilot study was maintained, except that now, following access to fluids in the individual drinking cages, subjects received $30-\mathrm{min}$ access to water in the home cages after they returned to the colony room. This supplementary drinking was given at the same time of day for a given group, ranging from 1000 to $1200 \mathrm{~h}$.

Subjects were first given access to water in the drinking boxes for $20 \mathrm{~min}$ on Day 1. Thereafter all sessions were of 10 -min duration. On Days 3-6, half the subjects in each home-cage squad of 8 rats were given water and half $0.2 \%$ saccharin solution. Throughout the experiment, the position of drinking bottles alternated between left and right to hinder the development of position preferences. This was counterbalanced within each group, as was the position of the bottles on choice tests. On Day 7, all subjects were given a choice test between water and $0.2 \%$ saccharin.

On Day 8 , subjects were given 10 -min water in the drinking chambers, followed by two-bottle tests between water and $\mathrm{HCl}$ on Days 9 and 10 . Since exposure to this taste had already been given in the pilot experiment, neophobia could not be assessed. Also, because of habituation due to the prior exposure, the sour solution used on these 2 days was $0.015 \mathrm{M} \mathrm{HCl}$.

On Days 11 and 12, a two-bottle test was given between water and $5.2 \mathrm{mg} / \mathrm{L}$ quinine sulphate monohydrate (Dess, Chapman, \& Minor, 1988).

\section{Results}

This experiment demonstrated strong neophobia to saccharin in all groups, without any indication that this might be affected by sex or strain differences. In contrast, when tested with the already familiar $\mathrm{HCl}$ solution, females were somewhat more tolerant than males and, when tested with the novel bitter solution, albinos were somewhat more tolerant than hooded rats.

Over the first 2 days males drank more water than females $[F(1,56)=84.94, p<.01]$, and hooded rats more than albinos $[F(1,56)=5.014, p<.05]$. The sex difference presumably resulted mainly from body weight differences. This could not account for the strain difference, since the hooded animals weighed somewhat less than the albinos. However, greater water intake is consistent with the greater food intake, greater defecation, and higher metabolic rate found in the hooded rats.

A measure of neophobia toward saccharin was required that would not be confounded with the group differences in absolute intakes shown in Figure 6. This was achieved by calculating each subject's intake of saccharin or water on Day 3 as a percentage of its intake of the same fluid on Day 6, by which time neophobia should have decreased very considerably. A 3-way ANOVA was applied to these scores, with sex, strain, and fluid (i.e., water vs. saccharin) as factors. This failed to detect main effects of either sex or strain (both $F \mathbf{S}<1$ ), whereas there was a significant effect of test fluid $[F(1,56)=33.37, p<.01]$, indicating neophobia for saccharin. No interactions were detected.

Data from the two-bottle test between saccharin and water given on Day 7 were converted into percent preferences for saccharin. The same 3-way ANOVA applied to 

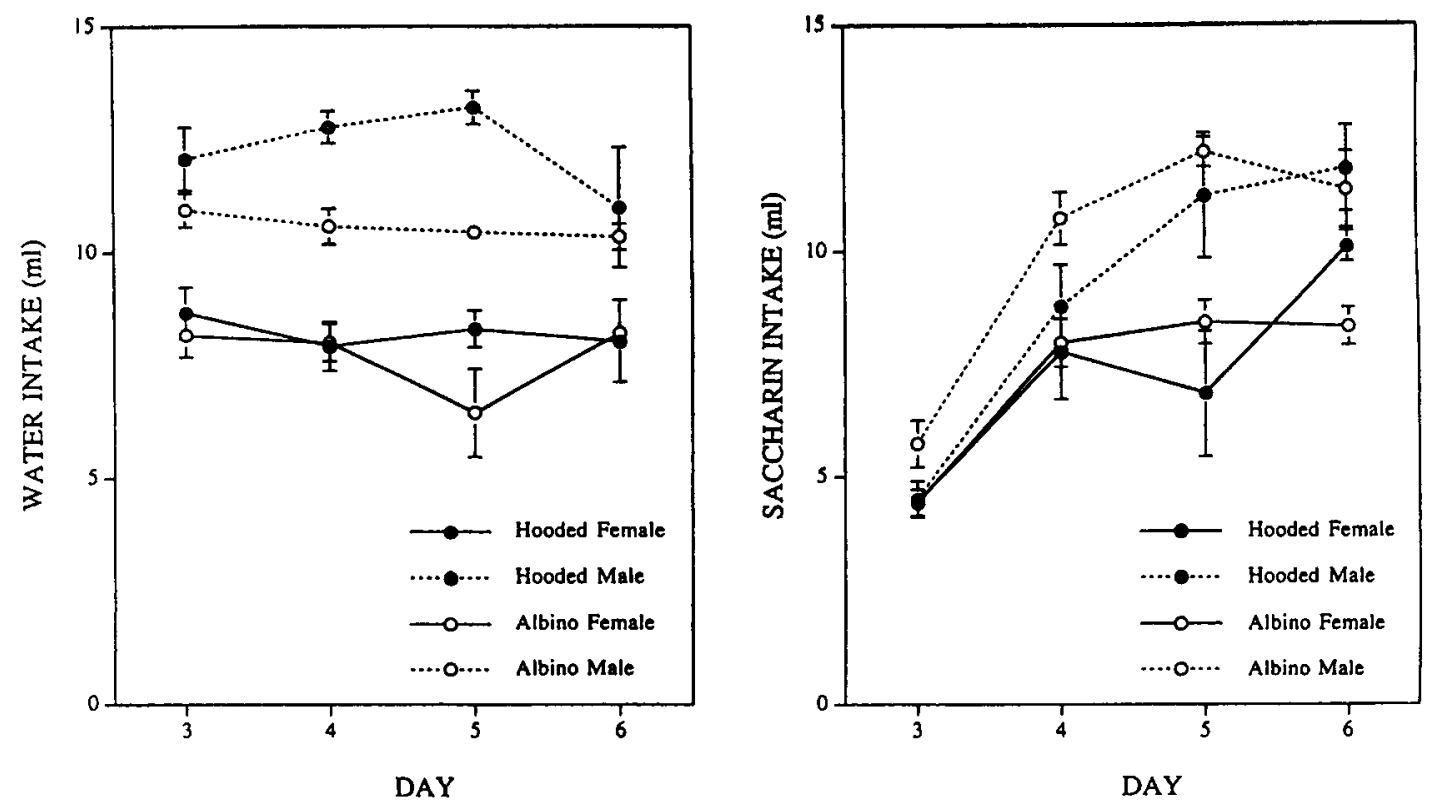

Figure 6. Experiment 4: Saccharin neophobia. Mean intake, plus standard errors of the mean, in four 10-min drinking sessions for the four groups $(n=8)$ given water (left panel) and in the four groups $(n=8)$ given $0.2 \%$ saccharin solution (right panel).

these scores, shown in Figure 7, indicated that subjects previously given saccharin on Days 3-6 had substantially higher preferences than those for which it was novel $[F(1,56)=85.51, p<.0001]$, thus providing an additional measure of neophobia to saccharin. There were no effects of sex or strain, nor were there any interactions $($ all $F \mathrm{~s}<1)$.

Results from the two-bottle tests between sour $(0.015 \mathrm{M}$ $\mathrm{HCl}$ ) and water are shown on the left side of Figure 8. Higher preferences for sour in females confirmed the indication from the pilot study. A 2-way ANOVA of these data revealed a significant main effect of $\operatorname{sex}[F(1,56)=$ $5.11, p<.05]$, but no effect of strain $(p>.10)$ or interaction $(F<1)$. Preference scores from the final two-bottle tests on Days 11 and 12 between bitter (quinine sulphate) and water are shown on the right side of Figure 8. In this case, the same analysis revealed an effect of strain, with higher preferences for bitter shown by the albinos $[F(1,56)=5.32, p<.05]$, but no sex difference $(p>.1)$, and again no interaction $(F<1)$.

\section{Discussion}

Overall there was little from this experiment to indicate that hooded and albino rats differ greatly in their responses to tastes. Under conditions that revealed a robust neophobic response to saccharin, as indicated both by within-subjects single bottle measures and by betweensubjects choice tests, no strain or sex differences were detected. Furthermore, once such neophobia had habituated, there was also no indication of strain or sex differences in preference for saccharin. Thus, these results fail to replicate Nachman's (1959) finding of greater preference for saccharin in hooded rats. This probably reflects the use of a different stock of rats and suggests that Nachman's finding may not be a general one. Furthermore, whatever genetic factor underlies the individual variation in acceptability of saccharin studied by Bartoshuk (e.g., 1979), it does not appear to have different prevalence across hoodeds and albinos in the Wistar strains used in the present study.

Since the more extensive examination of saccharin intakes failed to detect any strain or sex differences, the patterns of results obtained with hydrochloric acid, which was already familiar, and quinine, which was novel, are unlikely to reflect general differences in taste neophobia. Given the evidence for stress-induced finickiness (e.g., Dess \& Chapman, 1990), it is possible that the results shown in Figure 8 were influenced by differential response to the stress of, say, handling and transport prior to a session. However, one might expect these to have been seen earlier, when saccharin was given, since such finickiness has been reported for saccharin as well as quinine (Dess, 1992). Perhaps all that can be concluded from these data is that generalization from results obtained with one aversive taste (e.g., bitter) to another aversive taste (e.g., sour) should be made with caution.

Over Experiments 1-4, the most consistent ways in which hooded and albino rats differed were in metabolism and emotional reactivity. Although hooded rats tended to show more fear toward a new environment than albinos, there was no indication from Experiment 4 that they differed in neophobia toward saccharin. The only strain difference in taste reactivity detected in this experiment was the lower preference for quinine shown by the hooded rats. With regard to the learning tasks used in the follow- 


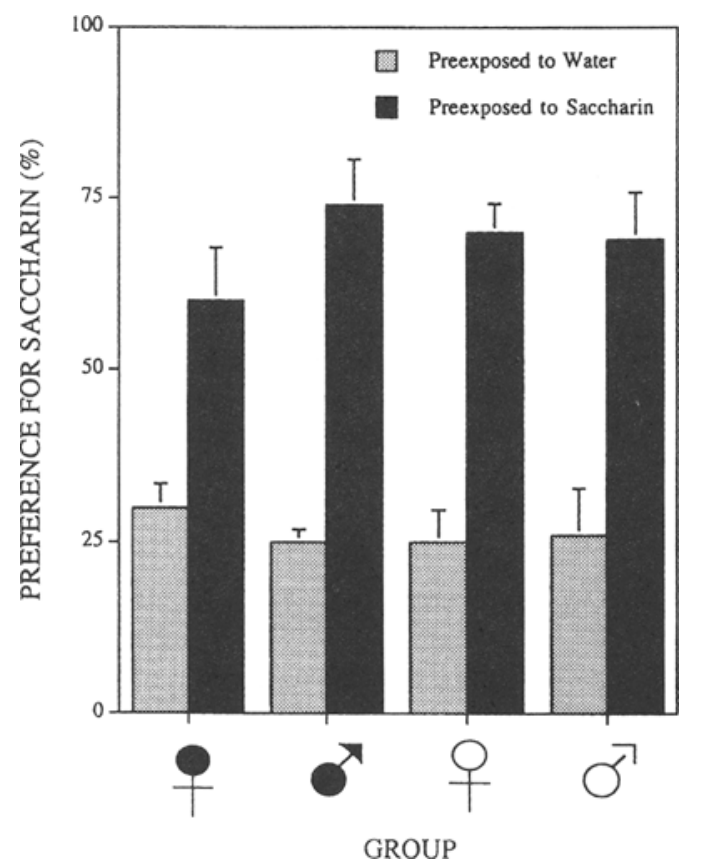

Figure 7. Experiment 4: Saccharin preference. Mean percent preference, plus standard errors of the mean, for $0.2 \%$ saccharin in the $10-\mathrm{min}$, two-bottle choice test between saccharin and water, comparing groups given saccharin for the four previous sessions $(n=8)$ with those for whom saccharin was novel on test $(n=8)$. Filled symbols indicate data from the hooded groups and open symbols those from the albino groups.

ing experiments, the results of these initial experiments suggest that, where emotional reactivity or metabolism is important, performance is likely to be affected by strain, but for those involving tastes, strain is likely to make little difference.

\section{Experiment 5 Instrumental Conditioning}

This experiment compared the four groups on acquisition and extinction of food-reinforced leverpressing. In addition, it examined the influence of motivational factors on responding in extinction. Under some conditions, rats that have acquired an instrumental response when food deprived and then been given unrestricted access to food prior to an extinction test (low-deprivation condition) produce as many responses in extinction as rats maintained on restricted feeding throughout (high-deprivation condition). This effect is currently of considerable interest in relation to theories of incentive learning (see, e.g., Dickinson, 1997; Dickinson \& Balleine, 1994; Dickinson, Balleine, Watt, Gonzalez, \& Boakes, 1995). In order to test whether strain differences might be important in such research, the factorial design commonly used in these experiments (see, e.g., Balleine, 1992) was also used here. Within each group half the rats were given prior experience of food pellets when not deprived of food (pre- exposure condition) and the remainder had no experience of food pellets (no preexposure) prior to magazine training under high deprivation. Following a limited amount of leverpress training under high deprivation, half the rats were tested in extinction when still on food deprivation (high-deprivation condition) and half after being returned to unrestricted food (low-deprivation condition). This generated four conditions: pre-high, pre-low, no-high, and no-low. From Balleine (1992), one would expect comparable responding in extinction in the prehigh, no-high, and no-low groups, with reduced responding in the pre-low group. Such a result for this last group would indicate that these rats, but not those in the nolow group, had learned that pellets are of low incentive value in the absence of food deprivation. Previous research of this kind has used only hooded rats. The present experiment tested whether such motivational factors in instrumental behavior are sensitive to strain differences.

\section{Method}

Subjects. Within each strain-sex squad equal numbers of subjects were arbitrarily allocated to the four conditions $(n=8)$. They had unrestricted access to water in their home cages, while access to food was varied as described below.

Apparatus. Eight conditioning chambers, $30 \times 24 \times 30 \mathrm{~cm}$, contained grid floors and sheet aluminum end walls and ceiling, with Plexiglas side walls. A 4.2-cm-wide lever, projecting $2 \mathrm{~cm}$ into the chamber, was mounted on the front wall at a height of $4.8 \mathrm{~cm}$ above the floor, with its center $3.5 \mathrm{~cm}$ from the left wall. Forty-fivemilligram Noyes pellets could be delivered to an open tray mounted centrally in the front wall. The distance from the right-hand edge of the lever to the nearest edge of the food tray was $5 \mathrm{~cm}$. The chambers were enclosed within wooden cubicles, which were diffusely lit and fitted with extractor fans.

Preexposure to the food pellets was given when the rats were placed in buckets made of translucent white polyurethane, with $30-\mathrm{cm}$ diameter circular cross section and heights ranging from 26 to $40 \mathrm{~cm}$.

Procedure. All daily sessions were of 30-min duration, except where noted below. During the initial preexposure phase, subjects had unrestricted access to food and water. On 3 successive days all subjects were placed in the buckets, where 50 food pellets were available in a petri dish for subjects in the preexposure condition and only an empty dish for the no-preexposure condition. Following the third session, food was removed from the home cages and subjects were maintained on 22.5 -h food deprivation for the instrumental training phase that followed. This began with two sessions of magazine training in the absence of the levers, when pellets were delivered on a random time (RT) 60-sec schedule. The levers were inserted for the remaining sessions. Four sessions of leverpress training were given in which responses were reinforced on a random interval (RI) 2-sec schedule in the first session, on a RI 15 -sec in the second, and on a RI 30-sec schedule for the last two training sessions. Sessions were terminated if a subject received 30 pellets before 30 min elapsed. To accelerate acquisition, a small amount of Blu-tac and crumbled pellet was placed on the levers in the first session. All subjects were given 1.5-h access to food in their home cages following the two magazine training and the first three leverpress sessions.

Following four sessions of leverpress training, rats in the pre-low and no-low conditions were returned to unrestricted access to food in their home cages for the remainder of the experiment, while the others remained on the 22.5 -h feeding schedule. On the following day an extinction test was given, when no pellets were delivered for leverpressing. In the final reacquisition session, leverpressing was again reinforced on a RI $30-$ sec schedule. 

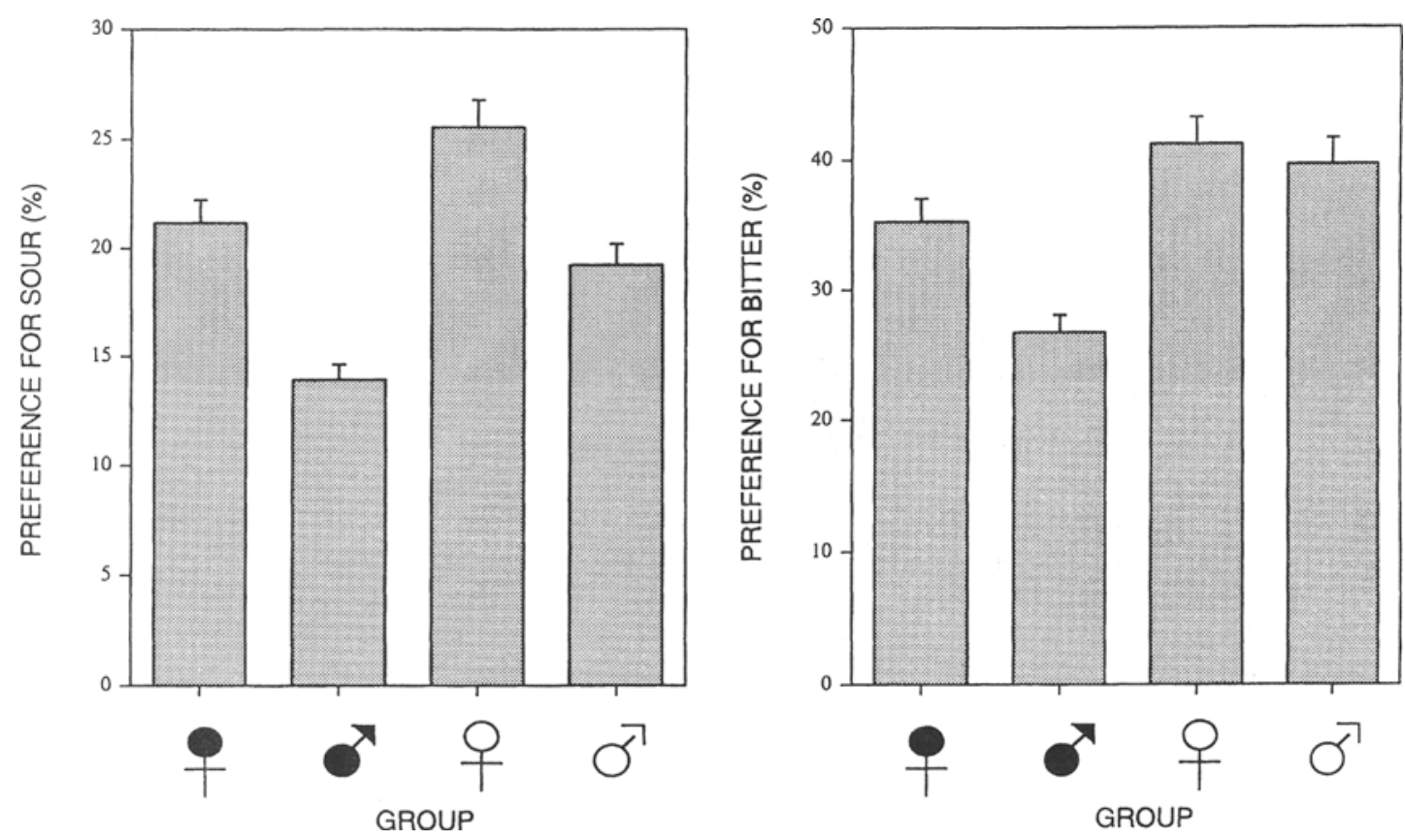

Figure 8. Experiment 4: Sour and bitter preferences. Mean percent preferences, plus standard errors of the mean, for sour over two 10-min choice tests between $0.015 \mathrm{M} \mathrm{HCl}$ and water (left panel) and for bitter over two 10-min choice tests between $5.2 \mathrm{mg} /$ liter quinine sulphate and water (right panel). Filled symbols indicate data from the hooded groups and open symbols those from the albino groups ( $n=16$ for all groups).

\section{Results}

The main finding from this experiment was that responding in extinction was directly affected by an animal's deprivation state (low vs. high), independently of whether it had previously experienced the food pellets when nondeprived (pre vs. no). Only limited strain and sex differences in leverpressing were detected, as detailed below.

During the pretraining phase, a marked strain effect was obtained. Albino rats ate virtually all the 50 pellets on all three sessions, whereas the hooded rats ate very few on the first session-averaging 14 pellets-but then increased their consumption over the next two sessions, consuming an average of 35 pellets on the third and final session. Number of pellets eaten per session was analyzed by a mixed ANOVA, with strain and sex as independent factors and session as a repeated measure. This revealed a main effect of strain $[F(1,28)=32.71, p<.01]$, a main effect of session $[F(2,56)=21.51, p<.01]$, and an interaction between strain and session $[F(2,56)=14.67, p<.01]$, which confirmed that the strain difference decreased over sessions. There was no effect of sex. Thus, these data indicated greater neophobia in hooded than in albino rats.

No systematic differences were observed in the acquisition of leverpressing, which was highly variable within the squads (all $F \mathrm{~S}<1$ ). Rates of leverpressing in the session prior to the extinction test were examined in an ANOVA with four independent factors: strain, sex, preexposure, and deprivation level. The only significant ef- fect was of sex: Males responded at a higher rate than females $[F(1,48)=8.343, p<.01]$.

The results of responding during extinction are shown in Figure 9. It may be seen that the male hooded rats and both sexes of albinos displayed the same pattern: higher levels of responding for subjects tested under high deprivation than for those tested under low deprivation. In none of the four groups was the result reported by Balleine (1992) evident-that is, lower responding in the pre-low than in the no-low subjects. In fact, the opposite tendency was seen in the exceptional group, the hooded females. The four-factor ANOVA that had been applied to responding in the final training session was carried out on these extinction data. This revealed an effect of strain, whereby hooded rats made more responses than albinos $[F(1,48)=$ $8.925, p<.005]$, and an effect of deprivation, whereby high deprivation produced more responding than low deprivation $[F(1,48)=21.78, p<.001]$. No other main effect or interaction was significant. Notably there was no sign of the interaction between preexposure and deprivation $(F<1)$ found by Balleine (1992; Dickinson et al., 1995).

A similar analysis of responding during the final reacquisition session again revealed a main effect of deprivation $[F(1,48)=76.50, p<.001]$, with greater responding under high than under low deprivation. In this session there was an effect of preexposure for the first time $[F(1,48)=9.438, p<.01]$, with preexposed subjects responding more than the nonpreexposed subjects. As in 


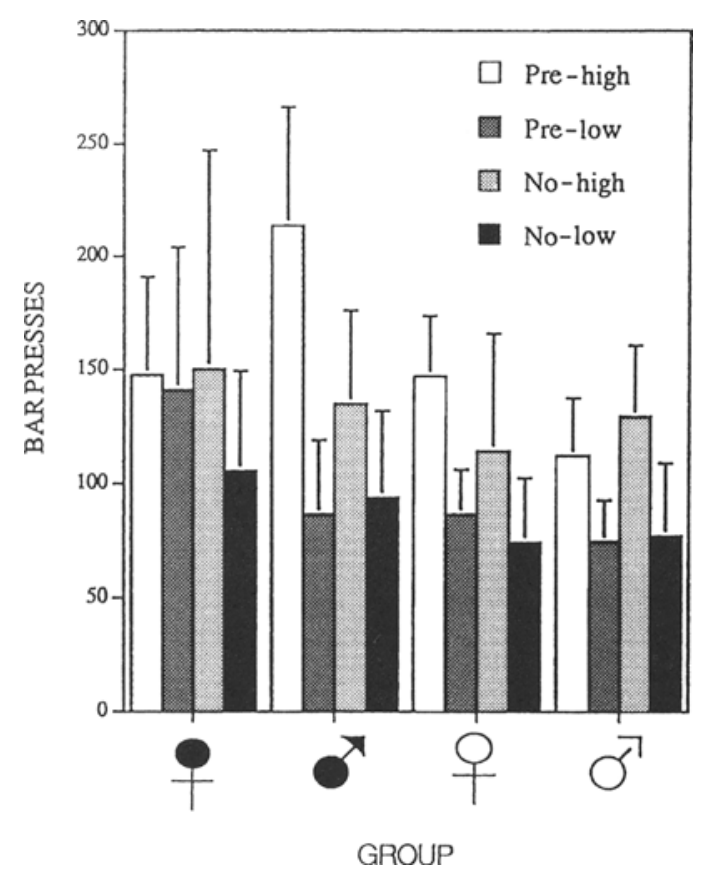

Figure 9. Experiment 5: Extinction of leverpressing. Number of responses during the 30-min extinction session. Open symbols indicate albinos and filled symbols hooded rats. Pre, groups that were preexposed to food pellets when in a low-deprivation state prior to leverpress training under high deprivation. High, groups that were maintained on the 22.5-h food schedule during the extinction test; Low, groups that had been returned to unrestricted food in the home cages prior to the test ( $n=16$ per group).

extinction, hooded rats responded more than albinos $[F(1,48)=8.652, p<.01]$, and, as in the final training session, males responded more than females $[F(1,48)=$ $12.19, p<.01]$. The only significant interaction detected was between strain, sex, and preexposure $[F(1,48)=$ $4.147, p<.05]$; this appeared to reflect a greater enhancement of responding in the male hooded rats by preexposure to the pellets, an effect that was small or nonexistent in the other three groups.

\section{Discussion}

In terms of strain and sex comparisons, it was remarkable how little the groups differed from each other. The most obvious strain difference was the greater neophobia shown by the hooded rats during initial pretraining with the food pellets. This contrasts with the failure to find any strain differences in neophobia toward a $0.2 \%$ saccharin solution in Experiment 4. What may have been a crucial difference between the two experiments is that the saccharin was presented in a context that was already highly familiar, whereas here the food pellets were first presented in a context that was also novel. Given the greater anxiety displayed by hooded rats in the open field of Experiment 1 and on the elevated plus maze of Experiment 2 , it seems likely that their lower intake of pel- lets in the present preexposure phase reflected a greater impact of the novelty of the buckets in suppressing eating, rather than neophobia toward the food pellets. The low deprivation state of the present subjects during pretraining may also have played a role. In Experiment 4, saccharin was given when the subjects were on a stringent water deprivation schedule. It may be that strain and other differences in neophobia are more easily detected when subjects are in a low deprivation state.

The only other strain difference that emerged was the higher rate of leverpressing shown by the hooded rats during extinction and reacquisition. Also, males were found to respond at a higher rate than females. Both findings contrast with sex and strain differences in activity found in the earlier experiments. In terms of wheel running, ambulation in an open field, and activity in a confined space, females were more active than males and, in general, albinos were more active than hoodeds. Thus, the results of Experiments 1 and 3 did not predict the differences in leverpressing obtained here.

The extinction test failed to detect an effect of preexposure of the kind reported from the similar experiment by Balleine (1992). This may be because of the configuration of the conditioning chambers. A preexposure effect is more likely to be found when there is a flap over the magazine aperture (Balleine, Garner, Gonzalez, \& Dickinson, 1995). The chamber used here contained no such flap. The present outcome is consistent with findings by Rescorla (1992) and inconsistent with incentive learning theory (e.g., Dickinson \& Balleine, 1994).

\section{Experiment 6 Latent Inhibition in Appetitive Pavlovian Conditioning}

When a stimulus is repeatedly paired with delivery of a response-independent appetitive reinforcer, typically the rate at which a rat approaches the site of the reinforcer during the signal-goal tracking-steadily increases (Boakes, 1977; Holland, 1977). Acquisition of such classically conditioned behavior is more rapid when the stimulus is novel; that is, a latent inhibition effect is found with this preparation (Channell \& Hall, 1983). The present experiment was designed to test for strain or sex differences both in acquisition of such behavior and in susceptibility to latent inhibition. Using a within-subjects design, each rat was trained with two sounds predicting delivery of the reinforcer, one to which it had been preexposed and one that was novel (Killcross, Dickinson, \& Robbins, 1994). To avoid possible differential effects of motivational factors on strain or sex differences, no deprivation schedule was employed. The reinforcer used, $30 \%$ sucrose solution, was expected to promote acquisition within a single session by nondeprived rats.

\section{Method}

Subjects. Only half the rats were used in this experiment, so that there were four 8-rat squads, housed together in same-sex and 
same-strain groups: male and female albinos, and male and female hooded rats. They had unrestricted access to food and water in their home cages throughout the experiment.

Apparatus. Four identical conditioning chambers measured 30 $\times 24 \times 50 \mathrm{~cm}$. The end walls and ceiling were made of aluminum sheet and the side walls of Plexiglas. The floor consisted of a grid of steel rods. In the front wall was a central recessed aperture where sucrose could be presented in a $0.1-\mathrm{ml}$ dipper cup. A Sonalert tone generator mounted centrally on this wall, $25 \mathrm{~cm}$ above the dipper aperture, was used to deliver a tone of $2.9 \mathrm{KHz}$. Above this a loudspeaker was used to deliver white noise. Presentations of both tone and noise were pulsed at $1 \mathrm{~Hz}$ with 0.5 -sec on time. A light and photocell mounted across the entrance to the magazine aperture were used to detect nosepokes.

Procedure. The procedure was modeled on one developed to study pharmacological factors in latent inhibition (McGregor \& Luscombe, 1994). Subjects were first given three 60 -min magazine training sessions, each containing 24 presentations of sucrose solution. Within each session, dipper access time was progressively reduced from 60 to $10 \mathrm{sec}$ and the interreinforcement interval was progressively increased from 20 to $340 \mathrm{sec}$. During the next two sessions there were no sucrose deliveries, and instead each subject was presented with one of the two 15-sec auditory stimuli for a total of 20 times in each session. Assignment of stimuli to this preexposure was counterbalanced across conditioning chambers and across squads. The interstimulus interval was variable with a mean of $3 \mathrm{~min}$ and a range of 1 to $5 \mathrm{~min}$.

The single acquisition session contained 20 trials in which a $25-$ $\mathrm{sec}$ auditory stimulus was followed $15 \mathrm{sec}$ after its onset by delivery of sucrose for $10 \mathrm{sec}$. The mean intertrial interval was $4.5 \mathrm{~min}$ with a range of 2 to $7 \mathrm{~min}$. Within each successive block of four trials, there were two presentations of the preexposed stimulus (pre-) and two of the stimulus that had not been presented previously (novel). For half the rats in each squad, the double alternation sequence, pre-novel-novel-pre, was used throughout the sessions, and for the other half the opposite sequence. Nosepokes were recorded for the $15 \mathrm{sec}$ before the start of the trial (pre-CS period) and during the $15 \mathrm{sec}$ prior to sucrose delivery (CS).

\section{Results}

Rates of nosepoking prior to presentation of a stimulus were uniformly low during the acquisition session. A preliminary analysis of these pre-CS data failed to reveal any differences on the basis of sex or strain. Progressively greater responding during the $\mathrm{CS}$ than during the pre-CS periods indicated that conditioning was obtained. This occurred more rapidly to the novel stimulus than to the one that had been preexposed; that is, latent inhibition was obtained. This description of the results was confirmed by a mixed ANOVA, with sex and strain as betweensubjects factors and preexposure versus novel, pre-CS versus CS, and trials as within-subjects factors. This revealed main effects of CS over pre-CS frequencies $[F(1,216)=179.4, p<.0001]$, of trials $[F(9,516)=7.379$, $p<.01]$, and an interaction between these two factors $[F(9,216)=27.32, p<.001]$, indicating that the difference between nosepoking during the $\mathrm{CS}$ and during the prior $15 \mathrm{sec}$ increased over the session. Latent inhibition was demonstrated by a simple interaction between preexposure and the pre-CS versus CS factor $[F(1,216)=$ $51.68, p<.0001]$, and by a triple interaction between these two factors and trials $[F(9,216)=2.143, p<.05]$, con-

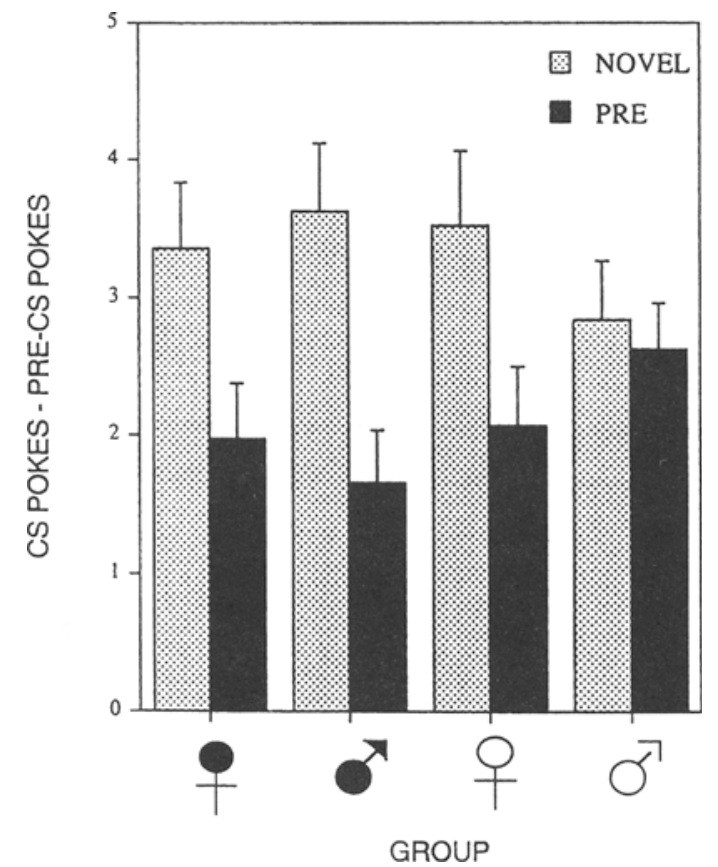

Figure 10. Experiment 6: Latent inhibition in appetitive classical conditioning. Differences between nosepokes during stimulus presentation (conditioned stimulus [CS]) and during the prior $15 \mathrm{sec}$ (pre-CS) averaged over the 10 presentations of the novel stimulus and the 10 presentations of the preexposed stimulus (pre). Open symbols indicate albino and filled symbols hooded rats.

firming that the rate of conditioning was more rapid to the novel stimulus.

The effects reported so far indicate that the procedure was an appropriate one for detecting possible sex or strain differences. In terms of overall rates of nosepoking, no significant strain or sex differences were detected [largest $F(1,216)=2.776, p>.10$ ]. Similarly, in terms of rate of conditioning independent of whether stimuli were preexposed or not, there was no indication of any difference between the squads: Strain and sex did not yield any significant interaction with either trials or the pre-CS versus CS difference (all $F \mathbf{s}<1$ ), and there was no interaction between all four factors [highest $F=1.354, p>$ .20]. The only significant effect of sex or strain was in the size of the latent inhibition effect, with the difference between nosepoking to the two stimuli most pronounced in the male hooded rats, as shown in Figure 10. This was confirmed by a triple interaction between strain, pre-CS versus $C S$, and preexposure $[F(1,216)=5.808, p<.05]$ and a quadruple interaction between sex, strain, pre-CS versus $C S$, and preexposure $[F(1,216)=6.834, p<.025]$.

To explore the nature of these interactions, a latent inhibition (LI) score was calculated for each subject as the CS-pre-CS difference score for the novel stimulus less the same difference score for the preexposed stimulus. When these scores were subjected to a $2 \times 2$ ANOVA, with strain 
and sex as factors, a significant effect of $\operatorname{strain}[F(1,24)=$ $5.81, p<.025]$ and significant interaction $[F(1,24)=$ $6.83, p<.025]$ were found, but no main effect of sex $(F<1)$. The nature of the interaction is shown in Figure 10, where CS-pre-CS differences for both the novel and preexposed stimuli are plotted for each of the four groups. It can be seen that the largest LI effect was obtained in the hooded males, contrasting with no LI effect in the albino males, whereas a moderate and equivalent LI effect was obtained from the two groups of females.

\section{Discussion}

As in the previous experiment, the most striking aspect of the present results was how little behavior differed across sex and strain. Whereas in Experiment 5, rates of leverpressing were higher for hooded rats and for males, no comparable differences in nosepoking were detected here. The fact that baseline rates of responding were comparable, plus the failure to detect any difference in acquisition of this classically conditioned behavior, provided an unexpected bonus with respect to the finding of differences in LI, which could otherwise have been compromised.

The greater sensitivity to preexposure of a stimulus in the hooded rats, and in particular in the hooded males, may have been specific to the use of auditory stimuli. It may also reflect the use of a within-subjects design, whereby the two auditory stimuli functioned identically as predictors of food. With this design an LI effect depends on good retrieval of the preexposed stimulus. It is possible that during retrieval albinos did not discriminate the sounds used here as CS as well as the hooded rats.

The present results appear inconsistent with those from a previous examination of strain differences in LI. Under conditions in which albino Wistar rats displayed an LI effect following preexposure to both auditory and visual stimuli, rats from a pigmented strain, Dark Agouti, showed the effect with visual stimuli but not when auditory stimuli were used (Weiner et al., 1998). Among the many differences between that study and the present experiment, one may be particularly important. Their experiments employed aversive motivation, with shock used as the reinforcing event in conditioned suppression and active avoidance procedures. Given the greater emotionality of hooded animals found in the present study, it is possible that the strain difference they obtained could have at least partly reflected greater unconditioned fear responses to auditory stimuli. Alternatively, the inconsistency between their results and the present finding may be because they compared rats from two different strains, with the consequence that genetic factors other than pigmentation are more likely to have been involved than in the present experiment.

\section{Experiment 7}

\section{Lithium-Based Taste and Context Aversions}

When a novel taste is followed by lithium chloride injection, an aversion to the taste is acquired, even with a considerable interval between the two events. Revusky (1971) found that such conditioning is retarded if another taste is experienced during this interval. In the present experiment, this serial interference paradigm was employed using a distinctive environmental context as the potentially interfering event. Thus, during the conditioning phase subjects were first given a novel taste, saline, and an hour later were placed in the target context and on removal injected with lithium. This allows the acquisition of both taste-nausea and context-nausea associations.

This procedure does not seem to have been employed before. However, prior conditioning of an environmental context can block acquisition of a taste aversion (Best, Brown, \& Sowell, 1984; Symonds \& Hall, 1997). This suggests that a context might function in a similar way to the interfering taste used by Revusky (1971). This procedure was employed because, since for all subjects both saline and target context were paired with lithium injection, it permitted detection of strain or sex differences in selectivity of taste-nausea conditioning. Conditioned context aversions were measured by testing for suppression of drinking of a familiar and palatable test fluid (sucrose) in the target context.

Following the group differences in LI found in the previous experiment, possible preexposure effects were also examined here. Subjects differed both as to whether they were given preexposure to the target context and as to whether they received intraperitoneal (i.p.) placebo injections before the start of conditioning. The latter factor was included because we had previously obtained some indication that injection cues might play a role in lithium-based aversion learning. It seemed possible that there could be strain or sex differences in sensitivity to such cues. Thus, within each strain-sex group a $2 \times 2$ design was used, with factors being preexposure to context versus novel context, and preexposure to injections (placebo) versus novel injection.

\section{Method}

Subjects. The experiment was run in two replications. The firstused animals ( $n=32$ ) that had not served in Experiment 6 and the second used animals that had $(n=32)$. The replications were identical except for the slight variation in preexposure noted below. Subjects continued to be housed communally in squads of 8 rats, with unrestricted access to food in their home cages and access to fluid restricted as described below.

Apparatus. Three sets of environmental contexts were employed The large white plastic buckets used as feeding boxes in Experiment 5 were modified so that $200-\mathrm{ml}$ drinking bottles could be mounted outside with their stainless steel spouts, containing two ball bearings, projecting inside, with the tips approximately $7 \mathrm{~cm}$ above the floor. These eight buckets served as the neutral context. The conditioning chambers used in Experiment 5 were also modified by placing a plain aluminum sheet over the grid floor, removing the levers, and covering three walls with chicken wire. The same drinking bottles could be inserted into the right-hand side of the front walls, with tips approximately $4.5 \mathrm{~cm}$ above the floor. The doors of the wooden shells containing these chambers were closed during experimental sessions, so that subjects were in complete darkness, and the fans provided masking noise. These eight chambers served as the target context. A third set of distinctive environ- 
ments consisted of eight rectangular cages, $21 \times 33 \times 18 \mathrm{~cm}$, made of transparent acrylic and with steel mesh ceilings into which the drinking bottles could be inserted with tips approximately $4 \mathrm{~cm}$ above the floor. These served as the control context. As with the buckets, there was visual but no auditory isolation between subjects in these drinking cages.

Procedure. During the pretraining phase (Days 1-14), subjects were adapted to drinking water in the neutral context for two periods each day, and preexposure to the target context and placebo injections was given to the appropriate groups. Water bottles were removed from the home cages on Day 0. On Days 1-4, all subjects received 10 -min access to water in the neutral context, were then returned to the colony room in their home cages, and $60 \mathrm{~min}$ later were given a further 20-min water in the neutral context. Thirty minutes after returning to the colony room for the second time, they were given 30-min access to water in their home cages. This schedule of supplementary drinking following a daily session was continued throughout the experiment. On Days 5-14, only 5-min access to water was given for the first drinking period, while the duration of the second drinking period remained at $20 \mathrm{~min}$ until Day 14 , when it was reduced to $10 \mathrm{~min}$.

Subjects in the placebo condition were given a total of six i.p. injections of $0.9 \%$ saline at $15 \mathrm{ml} / \mathrm{kg}$ immediately following the second drinking period on Days $3,5,7,9,11$, and 13. Subjects in the context preexposure condition were placed in the target context for the second drinking period of Days $6,8,10$, and 12 (first replication) or on Days $8,10,12$, and 14 (second replication), thus receiving a total of 80 and $70 \mathrm{~min}$, respectively, of context preexposure. On these days, the remaining subjects were given water in the control context.

The conditioning phase was identical for all subjects. It consisted of four cycles, each containing 3 days (Days 15-26). The 1st day of a cycle was the conditioning day or the first drinking period; 5-min access to $0.9 \%$ saline was given in the neutral context. Subjects were then returned in their home cages to the colony room. Sixty minutes later they were placed in the target context, where they received 10-min access to water. On removal from this context they were injected with $0.15 \mathrm{M}$ lithium chloride at $15 \mathrm{ml} / \mathrm{kg}$ and returned once more to the colony room. On the remaining 2 days of each cycle, the recovery days, subjects received water in the neutral context for $5 \mathrm{~min}$ in the first drinking period and $10 \mathrm{~min}$ in the second.

Extinction tests for an aversion to saline took place on Days 27, 29,31 , and 33. Each consisted of 5-min access to saline in the neutral context during the first drinking period. During the second period, subjects received 10 -min access to water, also in the neutral context. Extinction tests for an aversion to the target context took place on Days 28, 30, 32, and 34. Each consisted of 5-min access to water in the neutral context during the first drinking period, followed $60 \mathrm{~min}$ later by 10 -min access to $3 \%$ sucrose, as test fluid, in the target context.
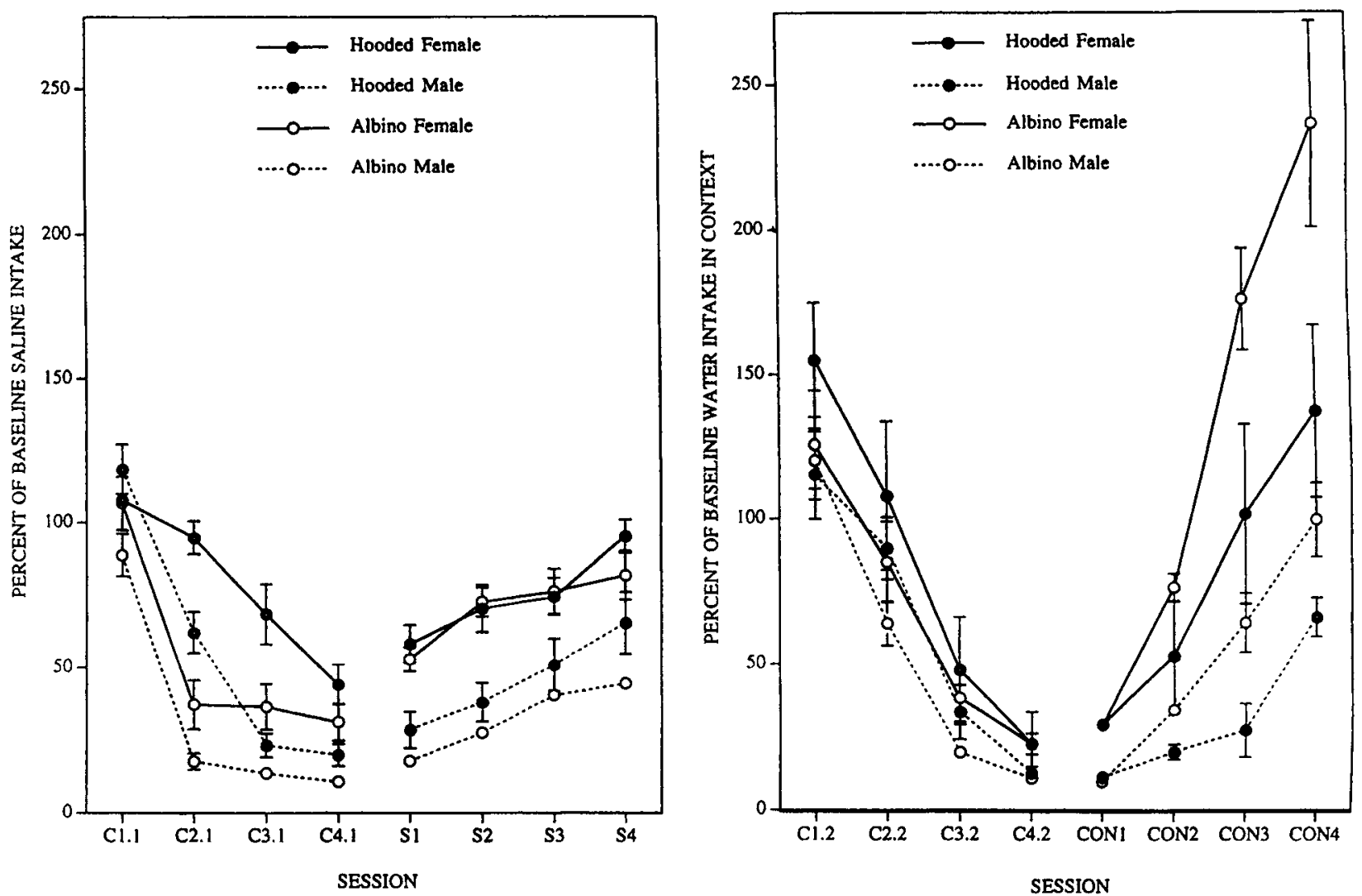

Figure 11. Experiment 7: Nausea-based conditioning of taste and context. Fluid intakes during conditioning sessions and subsequent extinction tests. Intakes, as percentages of baseline water intake, during the first of two daily drinking periods are presented in the left panel and intakes during the second period in the right panel. The left panel shows intakes in the neutral context when $0.9 \%$ saline was given for 5 min during both conditioning (C1.1, C2.1, etc.) and extinction (S1, S2, etc.). The right panel shows intakes in the target context during the 10-min drinking period that was given 60 min later. During the second period of conditioning sessions (Cl.2, C2.2, etc.), water was given. In the context test sessions (CON1, CON2, etc.), $3 \%$ sucrose was used as the test fluid ( $n=16$ per group). 


\section{Results}

The most important outcome from this experiment was that, whereas hooded rats showed somewhat weaker conditioning of a saline aversion than did albino rats, they showed a greater aversion to the target context, as indexed by suppression of drinking the test fluid, sucrose, in this context.

Water intakes during pretraining varied widely, with females drinking considerably less than males, as in Experiment 4 . Fluid intakes were therefore compared in terms of percentages of baseline drinking. Preliminary analyses failed to detect any effect of context or injection preexposure, nor did these factors interact with sex or strain. Furthermore, no effect of replication was detected, nor any interaction of this factor with any other. Consequently, all three factors were omitted from subsequent analyses.

Aversion to saline. As shown in the left-hand panel of Figure 11, saline intake decreased rapidly over the conditioning sessions, with males showing lower intakes than females, and albinos lower intakes and a more rapid decline than hooded rats. This was confirmed by a mixed ANOVA with sex and strain as between-subjects factors and sessions as a within-subjects factor. Conditioning was shown by a main effect of sessions $[F(1,180)=$ $129.26, p<.0001]$, lower intakes in males by a main effect of sex $[F(1,180)=27.438, p<.0001]$, and lower intakes in albinos by a main effect of strain $[F(1,180)=$ $27.404, p<.0001]$.

Intakes of saline during the extinction tests showed a clear separation on the basis of sex, but no significant strain difference. A similar ANOVA applied to these data revealed reliable extinction of the saline aversion, in that there was a main effect of sessions $[F(3,180)=17.583$, $p<.0001]$, as well as a main effect of $\operatorname{sex}[F(1,180)=$ $27.959, p<.0001]$. There was no main effect of strain $(F<1)$ and no interaction between sex and strain $(F=$ $1.085)$. There was also no indication of any effect of sex or strain on the rate of extinction (largest $F=1.416$, ps $>.20)$.

Aversion to the target context. Intakes in the target context of water during the second periods of conditioning sessions and of the test fluid, sucrose, during the second period of the context test sessions are shown in the right-hand panel of Figure 11. During conditioning sessions, the present serial procedure allows second-period drinking to be influenced by differences in first-period drinking, in that rats who drink a lot of fluid in the first period are likely an hour later to drink less than average. Since males drank less saline than females, and albinos less than hooded rats, in the first period of the conditioning sessions, one might expect to find the opposite pattern for water intake in the second period. This was not the observed result, and instead males again drank less than females. A mixed ANOVA of water intake during the conditioning sessions revealed a main effect of sessions $[F(1,180)=110.384, p<.0001]$ and a main effect of sex $[F(1,180)=7.226, p<.01]$, while the main effect of strain was marginal $[F(1,180)=2.955, p<.10]$. No interactions were significant (largest $F=1.601, p \mathrm{~s}>.10$ ).

Intakes of water in the target context may be a poor index of a conditioned aversion to this context, not only because of possible contamination by differences in firstperiod drinking, but more generally because animals may develop an aversion to water specific to the target context (Boakes, Westbrook, \& Barnes, 1992; Boakes, Westbrook, Elliott, \& Swinbourne, 1997). Consequently, a better indication of conditioned aversions to the target context is provided by suppression of drinking of sucrose during the context tests, when all animals drank water in the first period. As seen in the right panel of Figure 3, these tests revealed marked differences between the groups, with males drinking less sucrose and extinguishing more slowly than females, and hooded rats also drinking less sucrose and extinguishing more slowly than albinos. The same mixed ANOVA applied to these data showed an overall extinction effect, in that there was a main effect of sessions $[F(3,180)=61.252, p<.0001]$ and main effects of both sex $[F(1,180)=18.605, p<.0001]$ and strain $[F(1,180)=4.237, p<.05]$, but no interaction between sex and strain $(F<1)$. Differences in rates of extinction were confirmed by significant interactions between sessions and $\operatorname{sex}[F(1,180)=10.965, p<.0001]$ and between sessions and strain $[F(1,180)=7.533, p<.0001]$. There was no triple interaction between sessions, sex, and strain $(F=1.474, p>.20)$.

\section{Discussion}

The largest group effect found in this experiment was that of sex. Male rats showed stronger acquired aversions both to saline and to the target context. The simplest explanation of this difference is that, as in standard practice, when doses are calculated on the basis of body weight, lithium has a greater impact on males than on females. This cannot apply to the strain differences in conditioning obtained in the experiment, since here albinos tended to acquire stronger aversions to saline, but showed weaker aversions to the target context. This suggests that the target and neutral contexts were more discriminable for the hooded rats, thus allowing stronger conditioning of the target context.

The finding that albinos acquired a stronger saline aversion than hooded rats seems in conflict with the only previous comparison between hooded and albino rats in a taste aversion study. Dragoin (1971) reported that hooded male rats developed stronger aversions to hydrochloric acid solutions followed by various doses of cyclophosphamide than did albino rats. A number of factors suggest that this report may be misleading. First, it used pigmented rats of a different variety (Long Evans) from the albinos (Sprague-Dawley). Second, the acquisition and extinction data are reported only in terms of percentage of intake in the first conditioning session. Thus, the reported strain differences could have reflected differential neophobia or preference for the sour taste 
rather than differences in conditioning. Finally, Dragoin presented all solutions in the home cage whose associability with nausea is likely to have been low and similar across strains, in contrast to the present experiment, where associability of the target context did vary across strains.

No effect of preexposure to either the target context or injection cues was detected in any of the groups. This is consistent with subsequent experiments in our laboratory that also failed to detect reliable effects of giving rats experience of a target context prior to lithium-based conditioning (cf. Best \& Meachum, 1986) or of placebo injections designed to minimize the influence of injection cues. However, G. Hall (1997) has recently reported some evidence for context preexposure effects.

\section{Experiment 8 \\ Spatial Learning in a Radial Maze}

Experiments 5-7 employed extensive adaptation to the environments in which conditioning was carried out. Only subtle differences between hooded and albino rats were revealed. It seemed likely that more pronounced differences might emerge in a task that involved spatial learning. In such tasks the behavior of hooded rats was predicted to show greater neophobia, on the basis of their behavior in the open field test of Experiment 1, but to show greater ability to learn to discriminate between different places, on the basis of their superior ability to use distal visual cues (see, e.g., Tonkiss et al., 1992) and the greater sensitivity to contextual cues suggested by Experiment 7 .

The procedure selected was one that would allow group comparisons in terms of both simple spatial associative learning, or reference memory, and short-term, or working, memory for a recently visited arm in an eightarm radial maze. At the start of each session, food was available at the end of four arms, while the remaining four were never baited. Thus, optimal performance in each session would be to visit each baited arm once and never visit an unbaited arm (Olton \& Papas, 1979). Two kinds of error can be scored with this procedure: entry into an unbaited arm (failure of reference memory) and reentry into an arm that has already been visited on that session (failure of working memory).

\section{Method}

Subjects. The 8 rats from each group used in this experiment were those that had served in the first replication of Experiment 7. As in Experiment 6, and for the same reason of avoiding possible interactions with motivational factors, subjects were given unrestricted access to food and water in their home cages throughout the experiment, except for the 1 day noted below. Arms were baited with Kellogg's Coco Pops. Earlier in the study (Week 9), the rats had eaten this food very readily both when food deprived and when nondeprived, showing no sign of sex or strain differences in its palatability.

Apparatus. An elevated radial maze made of fiberboard sealed with polyurethane contained eight arms, length $70 \mathrm{~cm}$ and width
$9 \mathrm{~cm}$, at equal angular distances. Each arm contained hardwood walls, height $3 \mathrm{~cm}$, along each side and at the end, with a small cup placed at the end so that any food it contained was not visible until a rat reached the end of an arm. The octagonal central area measured $30 \mathrm{~cm}$ from one apex to the opposite one. There were no doors or any other obstruction to entry into any of the arms. The maze was mounted on a central pillar at a height of $65 \mathrm{~cm}$ and located toward the middle of a large laboratory space that contained a wide variety of extramaze cues.

Procedure. Rats received a session on alternate days throughout the experiment, with 4 rats from each group run each day. Complete counterbalancing was maintained across squads for session sequence, and thus for approximate time of day, and also for variations in the position of a rat when placed on the maze at the start of a session and for assignment of arms to be baited. Arms were wiped clean in between each session. Assignment of baited arms to subjects and the sequence in which they were run were also arranged to prevent the use of odor cues.

Pretraining was designed to ensure that all rats would readily approach the end of each arm and eat a Coco Pop whenever one was encountered. At the start of each session four Coco Pops were placed at approximately equal distances along each of the arms. A session ended when either all 32 Coco Pops had been eaten or $40 \mathrm{~min}$ had elapsed. Each rat received four such sessions.

In the training phase at the start of each session, one Coco Pop was placed in the cups at the end of four arms (e.g., these baited arms were 1,2,3, and 5 for the 1st rat in each group) and then the rat was placed in the center of the maze pointing in a prearranged direction that was systematically varied. A session was terminated as soon as the fourth Coco Pop was eaten, when the rat had made 17 visits to arm ends, or when $20 \mathrm{~min}$ had elapsed. A total of eight sessions were given in this phase. For each session a record was made of the time from placement in the maze until the first Coco Pop was eaten, the number of Coco Pops remaining at the end of the session, the number of boluses, and the sequence of arm entries until the first Coco Pop was eaten. An arm entry was defined as all four paws on an arm. Prior to Session 7, food was removed from the home cages so that subjects were deprived for approximately $21 \mathrm{~h}$ for this session only. This was to test whether food deprivation might have a differential effect on subjects' performance. They were returned to unrestricted food in the home cages following this session.

In a final rotation phase, four sessions using the same procedure were given with the maze rotated through $90^{\circ}$ from its position during training. This was to assess the possible role of intra- as opposed to extramaze cues.

\section{Results}

The major outcome of this experiment was that large sex and strain differences were found with respect to motivational factors, but very little with respect to spatial learning.

During pretraining, hooded rats took far longer to reach the ends of the arms and to eat the Coco Pops, and for both strains, males were slower than females. The neophobia displayed by the hooded males was such that 4 of these 8 subjects failed to eat all 32 Coco Pops within the 40-min time limit in two of the first three sessions, whereas only 2 hooded females were this slow, and none of the albinos were. However, by the fourth pretraining session, all subjects were eating all 32 Coco Pops within $40 \mathrm{~min}$. During this phase, the mean length of a session for females was $17.6 \mathrm{~min}$ for hooded rats and $5.7 \mathrm{~min}$ for albinos; for males, the means were $24.2 \mathrm{~min}$ for hooded 


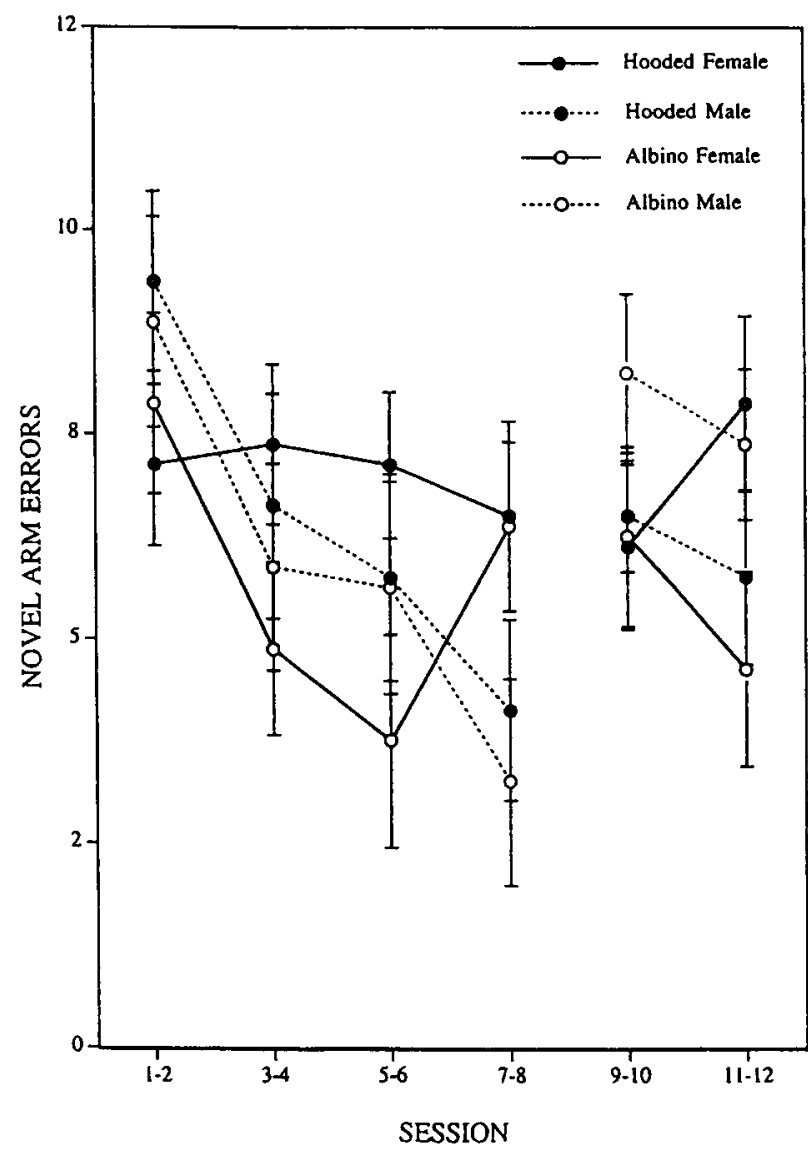

Figure 12. Experiment 8: Spatial learning in the radial maze. Mean number of entries to a previously unvisited arm (novel arm errors) prior to eating the first Coco Pop for the eight training sessions and the following four sessions when the maze was rotated through $90^{\circ}$ from its position during training ( $n=8$ per group).

rats and $7.8 \mathrm{~min}$ for albinos. An ANOVA of these scores revealed a main effect of $\operatorname{strain}[F(1,28)=53.9, p<.001]$ and a main effect of $\operatorname{sex}[F(1,28)=5.14, p<.05]$, but no interaction between these factors $[F(1,28)=1.36]$.

These differences during pretraining were similar to those found in the open field of Experiment 1. Over the five sessions of that experiment, there were also marked differences in bolus count, in that albinos did not defecate at all within the 2 -min test periods, whereas the hooded rats defecated a great deal, especially the males. The same was found during the present pretraining phase. Not a single albino defecated in any of the four sessions, whereas the hooded rats, particularly the males, defecated frequently. In relation to the time each hooded rat spent on the maze, the mean defecation rates were 7.34 boluses per hour for males and 3.91 boluses per hour for females $[t(8,8)=1.82, p<.05]$.

Measures of learning and memory. To assess reference memory - that is, learning not to enter the arms that had never contained food-novel arm errors were scored. This measure was the number of times in a session that a rat entered a previously unvisited arm prior to entering the arm in which it ate its first Coco Pop. This score included as an error entry into a baited arm followed by failure to reach the end or followed by ignoring the food. Thus, the maximum value was 8 . Acquisition functions based on these scores are shown in Figure 12, where it may be seen that these novel arm errors tended to decrease at first, but remained at a level well above zero, the score that would indicate perfect performance. Males produced lower scores than females. A mixed ANOVA of these scores over successive pairs of training sessions revealed a main effect of sessions $[F(3,84)=7.16, p<.002]$ and an interaction between sessions and sex $[F(1,84)=$ $2.96, p<.05]$. The only effect of food deprivation on performance on the seventh session was that subjects were more likely to eat a Coco Pop once one was found on this session than on the prior or following session.

Also shown in Figure 12 are novel arm errors for the four sessions following rotation of the maze through $90^{\circ}$ from its position during training. This had the effect of increasing error scores in subjects with a previously low score, notably the males, but little effect on subjects whose score had been relatively high. The number of novel arm errors on the final session of training, Session 8, was compared with the number on the first rotation session. Among the hooded rats, the mean for females decreased from 3.3 to 2.5 , and that for males increased from 1.1 to 4.0; among the albinos, mean errors increased from 2.9 to 4.1 in the females and 1.5 to 4.5 in the males. A mixed ANOVA was applied to novel arm errors for the two successive pairs of sessions following rotation. Unlike the outcome of the same analysis applied to training, there was no longer any effect of sessions or interaction involving this factor (largest $F=1.59$ ), and still no main effects of sex or strain, but a significant interaction between these factors was now revealed $[F(1,28)=4.85, p<.05]$. As shown in Figure 12, this interaction indicated that among hooded rats, the females made more errors than the males, but this was reversed for the albinos.

To assess short-term retention, or working memory, the tendency to return to an arm that had been previously visited was scored in terms of repetition errors. These were defined as the number of times prior to eating the first Coco Pop that a rat entered an arm that it had previously entered during that session. The most notable finding was how infrequent such errors were, even in the first few sessions, when many novel arm errors were made. Thus, in the training phase the mean number of repetition errors per session in the hooded rats was 0.78 for females and 0.50 for males, and in the albinos, it was 0.67 for females and 0.30 for males. There was no indication of any change in the frequency of these errors with training. An ANOVA of total repetition errors over the eight training sessions failed to reveal any effect of either strain or sex. 

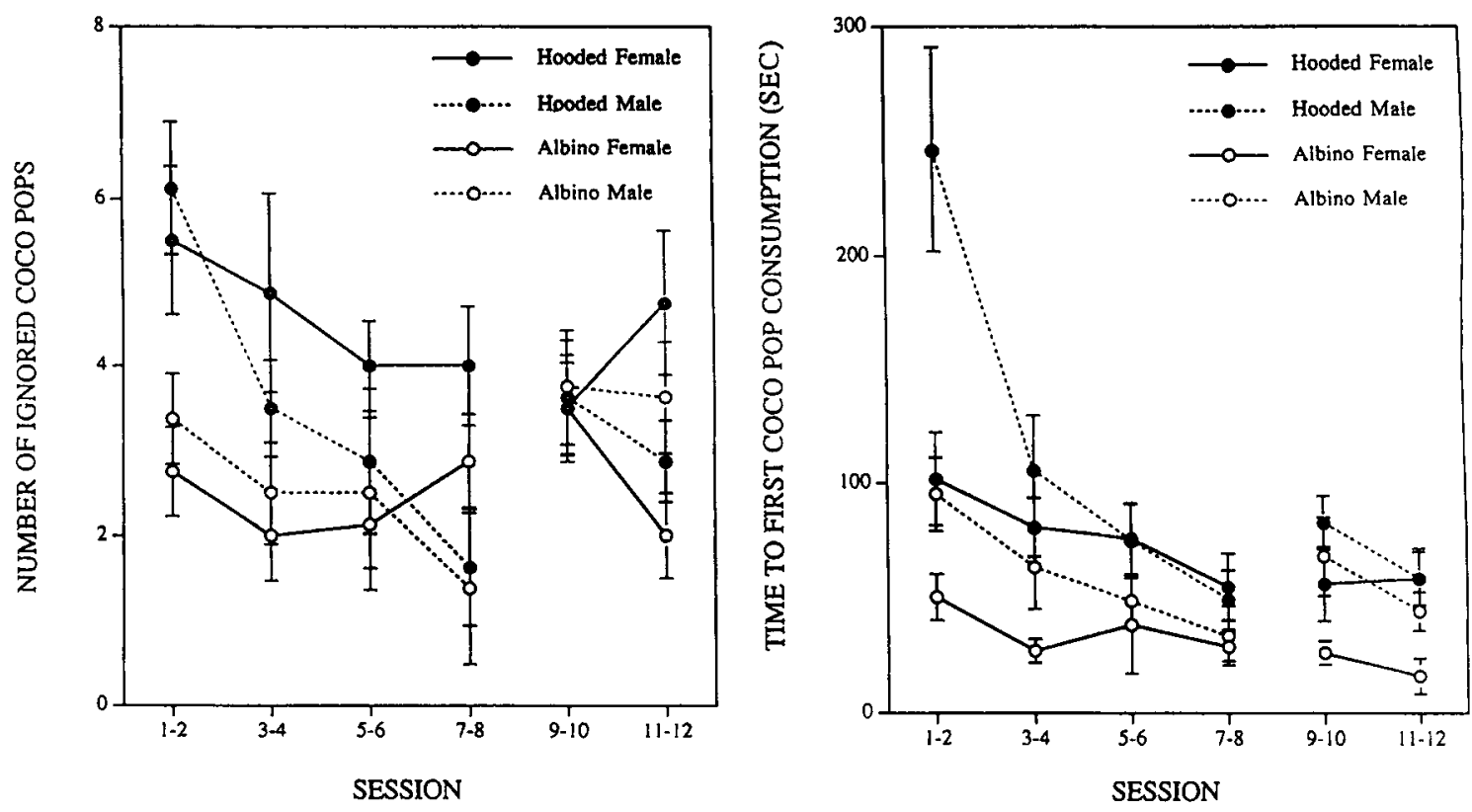

Figure 13. Experiment 8: Radial maze performance. Mean number of times per session when a rat failed to eat a Coco Pop it found at the end of an arm (left panel) and mean times (in seconds) until eating the first Coco Pop (right panel) ( $n=8$ per group).

A similar analysis of such errors over the four sessions following rotation of the maze also failed to reveal any group differences.

Measures of motivational factors. The above error scores were planned on the assumption that a rat's behavior would be influenced primarily by learning where Coco Pops were to be found and learning not to return to previously visited arms. Observation of the subjects' behavior suggested that this assumption was wrong. Particularly in the early part of a session, behavior did not appear to be guided so much by the food incentive as by a different kind of motivational control. The strong impression was conveyed that initially "exploring" the environment-or rather, "checking" it once the maze had become familiar-was a stronger motivational influence than food seeking. Such behavior on the part of rats given extensive exposure to a maze has been termed "patrolling" (Barnett, Dickson, Marples, \& Radha, 1978). The major difference between the groups appeared to lie in the relative strengths of competing motivational factors. Thus, the albinos appeared to spend little time patrolling the maze before settling into collecting the four Coco Pops, whereas for the hooded rats, the initial patrolling period was more extensive. Two measures of subjects' performance allowed examination of this possibility. One was the number of times that a rat reached the end of a baited arm but did not eat the Coco Pop. The other was the time until a rat ate its first Coco Pop in a session. As detailed below, both measures tended to confirm that hooded rats delayed eating more than albinos did.

As shown in the left-hand panel of Figure 13, initially hooded rats were more likely to ignore a Coco Pop than were albino rats. A mixed ANOVA applied to these scores from successive pairs of sessions during training revealed main effects of strain $[F(1,28)=13.6, p<.001]$ and of sessions $[F(3,84)=69.2, p<.001]$, but no other significant effects or interactions. Throughout the experiment, the hooded females tended to ignore on average the first four Coco Pops they encountered. Over the sessions following rotation of the maze, confirmation that this group differed from the others was obtained from a similar ANOVA that revealed an interaction between sex and strain $[F(1,28)=13.1, p<.05]$, but no other main effects or interactions.

The right-hand panel of Figure 13 shows the times from the start of a session to eating the first Coco Pop. Especially over the first few training sessions, this was the measure that revealed the most marked strain difference, with hooded rats much slower than albinos. Since these time scores were highly skewed, analysis was carried out on their reciprocals. For four consecutive pairs of training sessions, a mixed ANOVA revealed main effects of strain $[F(1,28)=13.7, p<.001]$ and of sessions $[F(3,84)=$ $9.23, p<.001]$. The only other significant effect was an interaction between these factors $[F(3,84)=4.54, p<$ $.01]$, indicating that the strain differences in speed decreased with training. Over the four sessions following rotation of the maze, significant strain and sex differences were found on this measure, with hooded still slower than albino rats $[F(1,28)=7.60, p<.05]$, males slower than females $[F(1,28)=6.38, p<.05]$, and a significant interaction between sex and strain $[F(1,28)=14.4, p<$ $.001]$, indicating that the sex difference was greater in the albino rats. 
Also examined were differences in the speed with which animals moved around the maze. Arguably this provides a better measure of the extent to which food-seeking behavior was suppressed by fear of, or distraction by, the environment. A rate measure for each session was obtained by dividing the number of arm entries by time. A mixed ANOVA of these scores over successive pairs of training sessions revealed that these speeds increased with training $[F(3,84)=15.2, p<.001]$, that the hooded rats were slower than the albinos $[F(1,28)=22.1, p<$ $.001]$, and that the males were slower than the females $[F(1,28)=6.21, p<.05]$, with no interaction between strain and sex $(F<1)$. An interaction between strain and sessions $[F(3,84)=4.66, p<.01]$ indicated that albino rats increased their speed with training more than did the hooded rats. Over the four sessions following rotation of the maze, there was a further slight increase in speed $[F(1,28)=5.32, p<5.32]$, but this no longer interacted with the other factors. However, albinos remained faster than hooded rats $[F(1,28)=30.2, p<.001]$ and females remained faster than males $[F(1,28)=20.4, p<.001]$, and there was now a strain $\times$ sex interaction $[F(1,28)=$ $12.0, p<.005$ ], indicating that the sex difference was largely confined to the albino rats.

\section{Discussion}

There was no indication from this experiment of any strain differences in learning where food was located. This was unexpected, since the disruption of performance resulting from rotation of the maze showed that all groups used extramaze cues to identify baited arms. The superior visual ability of hooded rats might be expected to enable them to learn about such cues more rapidly. However, since there was a very rich array of easily discriminable potential landmarks available, the poor vision of the albinos did not appear to have been a handicap in this setting. The use of less salient visual extramaze cues might have revealed superior spatial learning in the hooded animals.

As already noted, differences between the groups appeared to reflect motivational factors rather than learning. A raised and open environment of the kind used in this experiment can affect a rat's behavior in two ways. When novel, it can induce a high level of fear, as indexed here by defecation and suppression of locomotor activity. As it becomes increasingly familiar, it induces exploratory behavior that tends to be directed toward those parts of the environment less recently visited. Thus, a relatively unfamiliar arm of a maze elicits approach (see, e.g., Halliday, 1967). A sequence of such approaches would constitute patrolling. The present results suggest that sex and strain have different effects on these two factors, in a way that is entirely consistent with the results from the open field (Experiment 1 ) and plus-maze tests (Experiment 2). Those brief tests indicated greater neophobia in hooded than in albino rats, which was most marked in the hooded males. The much more extensive exposure provided by the present experiment allowed detection of differences in habituation of such neophobia based on sex and strain. When first exposed to such a situation, being hooded and being male both predict a high level of fear, as shown by the results reported for the pretraining phase. Since this fear habituates with repeated exposure, the strain difference becomes one of more extended patrolling on the part of hooded animals, as indexed by ignoring more food and moving more slowly before eating the first Coco Pop. In particular, an aspect of patrolling that hooded rats appeared to display far more frequently than albinos was to pause at the end of an arm, while sniffing and orienting toward various parts of the laboratory. This strain difference resembles the finding by Barnett et al. (1978) that patrolling of the arms of a large four-arm maze, in which a nest was provided in the middle as a living area, was more pronounced in wild rats than laboratory rats of an Agouti strain.

Gaffan and Davies $(1981,1982)$ reported data from two-arm mazes indicating the importance of spontaneous alternation, a tendency to enter the arm visited least recently, which was modified to only a limited extent by the acquisition of place-food associations. The present results are consistent with their analysis. Since there were eight arms, behavior could not be analyzed in terms of alternation. However, examination of the sequence of arms visited suggested a strong tendency toward entering an arm on the other side of the maze from the last one visited, even if this was not the one directly opposite. No subject adopted the response strategy of visiting adjacent arms in turn, but this is rarely displayed in a radial maze by rats beyond the age of around 13 weeks (Einon, 1980). The present subjects were over 30 weeks old when the experiment began. The tendency to enter an arm distant from the one just visited, but not exactly opposite, was present from the start of the experiment and is reflected in the low rate of repetition errors shown by all groups throughout the experiment. This meant that no evidence bearing on possible strain or sex differences in spatial working memory could be obtained, as originally intended. Clearly, none of the rats had any difficulty remembering where they had recently been.

\section{GENERAL DISCUSSION}

This set of experiments was primarily undertaken to enable researchers to make a more informed choice between pigmented and albino rats for use in experiments on learning. In general, the results suggest that it makes little difference for studies involving simple conditioning in an enclosed environment, providing that the subjects are first fully adapted. This requirement was met for instrumental conditioning in Experiment 5, appetitive classical conditioning in Experiment 6, and taste aversion learning in Experiment 7, in all of which strain differences were at best slight. When more subtle phenomena are of interest, the superior perceptual abilities of hooded animals may make them preferable. This is suggested by Experiment 6 , which required discrimination between two auditory stimuli for latent inhibition to be detectable and that found the effect to be largest in the 
hooded males. Experiment 7 suggested that hooded rats might also be more appropriate in those lithium-based conditioning experiments where the role of contextual factors is of interest.

When, as in Experiment 8, a procedure requires the use of an open environment, the greater sensitivity of hooded rats to distal cues (Tonkiss et al., 1992) may prove to be a disadvantage in that persistent patrolling behavior may interfere with performance based on place-food associations. This may, however, be less of a problem when animals are food deprived, a possibility not examined systematically in the present study. There are also some practical considerations in favor of albino subjects. Reflecting their lower metabolic rates and lower level of neophobia toward unfamiliar environmental stimuli, albino rats eat less, defecate less, and habituate more rapidly to a new setting.

A secondary aim of the study was to examine possible relationships between behavioral measures obtained in a variety of preparations. The one clear example of a consistent strain difference that appeared in a range of settings was neophobia, or emotionality in novel environments (Gray, 1987). Hooded rats were found to be more emotional than albinos in the open field of Experiment 1, the plus maze of Experiment 2, during pretraining prior to instrumental conditioning in Experiment 5, and, most markedly, on the radial maze in Experiment 8. This appears limited to environmental stimuli, since no strain difference in neophobia to saccharin was found in Experiment 3.

No such consistency was found across various measures potentially related to the Hullian concept of "general activity" (Bolles, 1975). Experiment 1 confirmed the well-established finding that females run faster in a wheel and make more line crossings in an open field than males. This difference was also found in terms of running speed around the radial maze, once considerable training had been given. By contrast, males were more active both in terms of the fine movements recorded in the metabolic chamber (Experiment 3 ) and in terms of leverpressing (Experiment 5), whereas no sex difference was found with nosepoking (Experiment 6). Overall, this lack of consistency with respect to sex differences in activity supports Bolles's conclusion that "general activity" is not a useful theoretical or empirical concept. As for strain differences on the various measures of activity, these were hardly seen, except when confounded with reactions to open environments, as in the open field and radial maze.

The present experiments failed to detect any strain or sex differences in measures of learning across a range of conditioning preparations. This is perhaps unsurprising in view of the notorious difficulty of obtaining consistent differences on such measures even when widely different species are compared (Macphail, 1982, 1987).

\section{REFERENCES}

AgGLETON, J. P. (1996). The ability of different strains of rats to acquire a visual nonmatching-to-sample task. Psychobiology, 24, 44-48.

Andrews, J. S., Jansen, J. H. M., Linders, S., Princen, A., \& BroekKAMP, C. L. E. (1995). Performance of four different rat strains in the autoshaping, two-object discrimination, and swim maze tests of learning and memory. Physiology \& Behavior, 57, 785-790.

Atrens, D. M., \& Menendez, J. A. (1993). Glucagon and the paraventricular hypothalamus: Modulation of energy balance. Brain Research, 630, 245-251.

BALLEINE, B. W. (1992). Instrumental performance following a shift in primary motivation depends on incentive learning. Journal of Experimental Psychology: Animal Behavior Processes, 18, 236-250.

Balleine, B. W., Garner, C., Gonzalez, F., \& Dickinson, A. (1995). Motivational control of heterogeneous instrumental chains. Journal of Experimental Psychology: Animal Behavior Processes, 21, 203-217.

Barnett, S. A., Dickson, R. G., Marples, T. G., \& Radha, E. (1978). Sequences of feeding, sampling and exploration by wild and laboratory rats. Behavioral Processes, 3, 29-43.

BarToshuK, L. M. (1979). Bitter taste of saccharin related to genetic ability to taste bitter substance 6- $n$-Propylthiouracil. Science, 205, 934-935.

Best, M. R., Brown, E. R., \& Sowell, M. K. (1984). Taste-mediated potentiation of noningestional stimuli in rats. Learning \& Motivation, 13, 244-258.

BEST, M. R., \& MEACHUM, C. L. (1986). The effects of stimulus preexposure on taste-mediated environmental conditioning: Potentiation and overshadowing. Animal Learning \& Behavior, 14, 1-5.

BIRCH, D., \& JACOBS, G. H. (1979). Spatial contrast sensitivity in albino and pigmented rats. Vision Research, 19, 933-937.

BOAKES, R. A. (1977). Performance on learning to associate a stimulus with positive reinforcement. In H. Davis \& H. M. B. Hurvitz (Eds.), Operant-Pavlovian interactions (pp. 67-97). Hillsdale, NJ: Erlbaum.

Boakes, R. A., Westbrook, R. F., \& Barnes, B. W. (1992). Potentiation by a taste of toxicosis-based context conditioning: Effects of varying the test fluid. Quarterly Journal of Experimental Psychology, 45B, 303-325.

Boakes, R. A., Westbrook, R. F., Elliott, M., \& Swinbourne, A. L. (1997). Context dependency of conditioned aversions to water and sweet tastes. Journal of Experimental Psychology: Animal Behavior Processes, 23, 56-67.

Bolles, R. C. (1975). Theory of motivation (2nd ed.). New York: Harper \& Row.

BROADHURST, P. L. (1960). Experiments in psychogenetics. In H. J. Eysenck (Ed.), Experiments in personality (Vol. 1, pp. 3-70). London: Routledge \& Kegan Paul.

Channell, S., \& Hall, G. (1983). Contextual effects in latent inhibition with an appetitive conditioning procedure. Animal Learning \& Behavior, 11, 67-74.

CreEl, D. J. (1980). Inappropriate use of albino animals as models in research. Pharmacology, Biochemistry \& Behavior, 12, 969-977.

DeFries, J. C., HegmanN, J. P., \& Weir, M. W. (1966). Open field behavior in mice: Evidence for a gene effect mediated by the visual system. Science, 154, 1577-1579.

DEss, N. K. (1992). Divergent responses to saccharin vs sucrose availability after stress in rats. Physiology \& Behavior, 52, 115-125.

Dess, N. K., \& Chapman, C. D. (1990). Individual differences in taste, body weight and depression in the "helplessness" model and in humans. Brain Research Bulletin, 24, 669-676.

Dess, N. K., Chapman, C. D., \& Minor, T. D. (1988). Inescapable shock increases finickiness about drinking quinine-adulterated water in rats. Learning \& Motivation, 19, 408-422.

Dickinson, A. (1997). Bolles' psychological syllogism. In M. E. Bouton \& M. S. Fanselow (Eds.), The functional behaviorism of Robert C. Bolles: Learning, motivation and cognition (pp. 345-367). Washington, DC: American Psychological Association.

Dickinson, A., \& Balleine, B. (1994). Motivational control of goaldirected action. Animal Learning \& Behavior, 22, 1-18.

Dickinson, A., Balleine, B., Watt, A., Gonzalez, F., \& Boakes, R. A. (1995). Motivational control after extended instrumental training. Animal Learning \& Behavior, 23, 197-206.

DRAGOIN, W. B. (1971). Conditioning and extinction of taste aversions with variations in the intensity of the CS and UCS in two strains of rats. Psychonomic Science, 22, 303-305.

EINON, D. (1980). Spatial memory and response strategies in rats: Age, sex and rearing differences in performance. Quarterly Journal of Experimental Psychology, 32, 473-489. 
Gaffan, E. A., \& Davies, J. (1981). The role of exploration in win-shift and win-stay performance on a radial maze. Learning \& Motivation, 12, 282-299.

GafFan, E. A., \& Davies, J. (1982). Reward, novelty and spontaneous alternation. Quarterly Journal of Experimental Psychology, 34B, 31 47.

GraY, J. A. (1987). The psychology of fear and stress. Cambridge: Cambridge University Press.

HaLL, C. S. (1936). Emotional behavior in the rat: III. The relationship between emotionality and ambulatory activity. Journal of Comparative Psychology, 22, 345-352.

HALL, G. (1997). Context aversion, Pavlovian conditioning, and the psychological side effects of chemotherapy. European Psychologist, 2 , 118-124.

Halliday, M. S. (1967). Exploratory behaviour in elevated and enclosed mazes. Quarterly Journal of Experimental Psychology, 19, 254-263.

Hamilton, L. W., \& Timmons, C. R. (1976). Sex differences in response to taste and postingestive consequences of sugar solutions. Physiology \& Behavior, 17, 221-225.

Holland, P. C. (1977). Conditioned stimulus as determinant of the form of the Pavlovian conditioned response. Journal of Experimental Psychology: Animal Behavior Processes, 3, 77-104.

Johnston, A. L., \& FiLE, S. E. (1991). Sex differences in animal tests of anxiety. Physiology \& Behavior, 49, 245-250.

Katz, R. J., \& Doyle, R. L. (1981). The albino locus and locomotor behaviour in the mouse: Studies using extended test intervals. Behavior Genetics, 11, 167-172.

Killcross, A. S., Dickinson, A., \& Robiins, T. W. (1994). Amphetamine-induced abolitions of latent inhibition are reinforcer mediated: Implications for animal models of schizophrenia. Psychopharmacology, 115, 185-195.

LASHIEY, K. S. (1930). The mechanism of vision: II. The comparative visual acuity of pigmented and albino rats. Journal of Genetic Psychology, 37, 481-484.

LuND, R. D., LuND, J. S., \& WiSE, R. P. (1974). The organization of retinal projection to the dorsal lateral geniculate in pigmented and albino rats. Journal of Comparative Neurology, 158, 383-404.

MACPHAIL, E. M. (1982). Brain and intelligence in vertebrates. Oxford: Oxford University Press, Clarendon Press.

MacPhalL, E. M. (1987). The comparative psychology of intelligence. Behavioral \& Brain Sciences, 10, 645-695.

MCGregor, I. S., \& LusCombe, G. M. (1994). The role of the 5HT2 re- ceptor in latent inhibition [Abstract]. Behavioral Pharmacology, 5 (Suppl. 1), 120

MistLberGER, R. E. (1993). Circadian food-anticipatory activity: Formal models and physiological mechanisms. Neuroscience \& Biobehavioral Reviews, 18, 171-195.

NACHMAN, M. (1959). The inheritance of saccharin preference. Journal of Comparative \& Physiological Psychology, 52, 451-457.

Olton, D. S., \& PAPAS, B. C. (1979). Spatial memory and hippocampal function. Neuropsychologia, 17, 669-682.

Overstreet, D. H., RezVANI, A. H., \& JanowsKy, D. S. (1992). Maudsley reactive and non-reactive rats differ only in some tasks reflecting emotionality. Physiology \& Behavior, 52, 149-152.

Pellow, S., Chopin, P., File, S. E., \& Briley, M. (1985). Validation of open:closed arm entries in an elevated plus-maze as a measure of anxiety in the rat. Journal of Neuroscience Methods, 14, 149-167.

Rescorla, R. A. (1992). Depression of an instrumental response by a single devaluation of its outcome. Quarterly Journal of Experimental Psychology, 44B, 123-136.

REVUSKY, S. (1971). The role of interference in association over a delay. In W. K. Honig \& P. H. R. James (Eds.), Animal memory (pp. 155213). New York: Academic Press.

SAunders, D. C. (1981). The Bethlem lines: Genetic selection for high and low rearing activity in rats. Behavior Genetics, 11, 491-502.

Symonds, M., \& HaLL, G. (1997). Contextual conditioning with lithiuminduced nausea as the US: Evidence from a blocking procedure. Learning \& Motivation, 28, 200-215.

Tonkiss, J., Shultz, P., \& Galler, J. R. (1992). Long-Evans and Sprague-Dawley rats differ in their spatial navigation performance during ontogeny and at maturity. Developmental Psychobiology, 25, 567-579.

Van der StaAy, F. J., Kerbusch, S., \& RaAiJmakers, W. (1990). Genetic correlations in validating emotionality. Behavior Genetics, 20, $51-62$.

WARD, R., \& LARIVIERE, H. (1980). An interactive effect of visual deprivation and pigmentation on the reactivity of mice to light. Behavior Genetics, 10, 419-424.

Weiner, I., Hairston, I., Shayit, M., Feldman, G., Joel, D., \& FelDON, J. (1998). Strain differences in latent inhibition. Psychobiology, 26, 57-64.

(Manuscript received August 4, 1998; revision accepted for publication August 26, 1999.) 